\title{
Approximation of H(div) with High-Order Optimal Finite Elements for Pyramids, Prisms and Hexahedra
}

\author{
Morgane Bergot ${ }^{\mathrm{a}}$, Marc Duruflé ${ }^{\mathrm{b}}$ \\ ${ }^{a}$ CALVI project team, INRIA Nancy-Grand Est, Strasbourg, FRANCE \\ morgane.bergot@inria.fr \\ ${ }^{b}$ BACCHUS project team, INRIA Bordeaux Sud-Ouest, Bordeaux, FRANCE \\ marc.durufle@inria.fr
}

\begin{abstract}
Classical facet elements do not provide an optimal rate of convergence of the numerical solution toward the solution of the exact problem in $H(d i v)$-norm for general unstructured meshes containing hexahedra and prisms. We propose two new families of high-order elements for hexahedra, triangular prisms and pyramids that recover the optimal convergence. These elements have compatible restrictions with each other, such that they can be used directly on general hybrid meshes. Moreover the $H(d i v)$ proposed spaces are completing the De Rham diagram with optimal elements previously constructed for $H^{1}$ and $H(\mathrm{curl})$ approximation. The obtained pyramidal elements are compared theoretically and numerically with other elements of the literature. Eventually, numerical results demonstrate the efficiency of the finite elements constructed.
\end{abstract}

Keywords: Facet Elements, High-order finite element, Pyramids, H(div) approximation, De Rham diagram

\section{Introduction}

The aim of this article is to build optimal $H(d i v)$-conforming elements in the same spirit as done in (1) and (2) for $H^{1}$ and $H(\mathrm{curl})$ conforming formulations. Finite elements for $H(\mathrm{div})$ formulations can be used for example to solve Stokes problem like in (3) or Darcy flow equation (see (4)). The other goal of this article is to complete the De Rham diagram for optimal elements introduced in (1) and (2) of an hybrid mesh (including hexahedra, tetrahedra, triangular prisms and pyramids).

A first family of finite elements for $H(d i v)$ formulations has been introduced by Nédélec for hexahedra and tetrahedra in (5), a second one being introduced in (6) for hexahedra, tetrahedra and triangular prisms. Nédélec's first family for $H(d i v)$ approximation is also known as Raviart-Thomas elements (7). However, when these elements are used on general unstructured meshes (especially hexahedral meshes), the interpolation error $E_{H(d i v)}$ is not optimal:

$$
E_{H(d i v)}=\|u-\pi u\|_{H(d i v)}=O\left(h^{\max (0, r-2)}\right)
$$

where the $H(d i v)$ norm is defined as

$$
\|u\|_{H(d i v)}^{2}=\|u\|_{2}^{2}+\|\operatorname{div} u\|_{2}^{2},
$$

$\pi$ being a projector from $H(d i v)$ on the discrete Raviart-Thomas space, $r$ the order of approximation and $h$ the mesh size. This sub-optimal convergence may be very problematic for lowest-order elements and unstructured meshes including highly distorted elements. The sub-optimal convergence is obtained when the elements of the mesh are not tending to affine elements when $h$ tends to 0 . Affine elements are elements such that the transformation $F$ linking reference elements (unit cube, unit prism, unit tetrahedron and symmetric pyramid) to elements of the mesh is affine. This case occurs if the element is only made of triangular faces (i.e. for tetrahedra), or when the quadrilateral faces are parallelograms. In (8), some numerical experiments illustrate the lack of consistency of lowest-order elements. The authors propose non-linear functions in order to obtain a convergent method. An another approach based on the splitting of an hexahedron in tetrahedra is proposed in (4). These two contributions seem difficult to extend to higher orders and the expression of basis functions on the reference element depend on the geometry. 
The aim of this paper is to construct high order elements for hexahedra, tetrahedra, prisms and pyramids ensuring an optimal estimate $O\left(h^{r}\right)$ in $H(d i v)$ norm. Such elements will be based on Piola transform, i.e. a basis function $u$ on the real element will be obtained as:

$$
u \circ F(\hat{x}, \hat{y}, \hat{z})=\frac{1}{|D F|} D F \hat{u}(\hat{x}, \hat{y}, \hat{z})
$$

where $(\hat{x}, \hat{y}, \hat{z})$ are the coordinates defined on the reference element $\hat{K}, D F$ is the jacobian matrix of transformation $F$ (which transforms $\hat{K}$ to the real element $K$ ), $|D F|$ its determinant. Moreover, these elements will be constructed such that basis functions on the reference element $\hat{u}$ will belong to finite element spaces $\hat{P}_{r}$ that do not depend on the geometry, that is to say they do not depend on the real element $K$. Such finite elements have been obtained for general quadrilateral elements in (9), and for lowest-order hexahedral elements in (10). In the theorem 3, these super-optimal spaces are detailed at any order for the four types of elements, the lowest order hexahedral space is the same as in (10). However, these spaces are not unique and not very practical to implement, optimal spaces will be detailed in the theorem 2 with more attractive properties. The dimensions of these spaces are very close to super-optimal ones.

To make the transition between triangular and quadrangular faces in a conformal hybrid mesh, the introduction of pyramids is needed (see (11)). Some pyramidal elements has been build by Nigam and Phillips, a first family in (12), a second one in (13). The optimal pyramidal space detailed in this paper will be compared to Nigam and Phillips spaces, and also to basis functions proposed in (14). Numerical experiments show that the optimal space require less degrees of freedom to reach a given accuracy.

We consider the following wave equation:

$$
\left\{\begin{array}{l}
-\frac{\omega^{2}}{\mu} u+-\nabla\left(\frac{1}{\rho} \operatorname{div} u\right)=f, \text { in } \Omega \\
u \cdot n=0, \text { on } \Gamma \\
\operatorname{div} u=-i \omega u \cdot n, \text { on } \Sigma
\end{array}\right.
$$

with $\omega$ the pulsation, $\rho, \mu$ physical coefficients related to the considered media. This simple equation enables us to study $H(d i v)$ finite elements in hybrid meshes. A Dirichlet condition is set on the inner boundary $\Gamma$ and an absorbing boundary condition is set on the outer boundary $\Sigma$. As a result, this boundary value problem is well-posed for any $\omega \in \mathbb{R}$. The variational formulation of the problem is equal to:

$$
\left\{\begin{array}{l}
\text { Find } \mathrm{u} \in H(\operatorname{div}, \Omega) \text { such that } \\
\forall v \in H(\operatorname{div}, \Omega), \quad-\omega^{2} \int_{\Omega} \frac{1}{\mu} u \cdot v d x+\int_{\Omega} \frac{1}{\rho} \operatorname{div} u \operatorname{div} v d x-i \omega \int_{\Sigma} u \cdot n v \cdot n d x=\int_{\Omega} f \cdot v d x
\end{array}\right.
$$

The outline of the paper is as follows:

- Section 2 is devoted to the construction of spaces for the four types of elements (hexahedron, tetrahedron, triangular prism and pyramid) as done in (1) and (2). A first set of "optimal" spaces is constructed in Section 2.2. To satisfy sharper conditions on the number of degrees of freedom, we construct a set of "super-optimal" spaces in Section 2.3. The trace of the two spaces is precised in Section 2.4 to verify the conformity at the interfaces.

- The stablity of the elements is treated in Section 3 where we prove that the sequence of the De Rham diagram is exact with the spaces of (1) (2) and the super-optimal spaces constructed.

- Quadrature formula used to get exact integrals whenever it is possible for the basis functions are presented in Section 4. When the quadrature formulas provide an approximate integration, it has been numerically observed that this approximation does not deteriorate the order of convergence of the finite element method.

- We present in Section 5 a space for pyramids compatible with the classical Nédélec's first family. 
- Hierarchical basis function are finally given in Section 6, for the optimal space for any type of element, for the super-optimal space and the hexahedron, and for the first family and the pyramid.

- Section 7 is devoted to the comparison of the new elements to elements found in the literature. A dispersion analysis and a study of the convergence are conducted for different kind of meshes. These numerical results show that optimal elements require generally less degrees of of freedom than other elements.

\section{2. $H($ div $)$-conforming Finite Element Spaces}

\subsection{Definition of Elementary Spaces}

Let us first introduce the following classical polynomial spaces:

\section{Definition 1.}

\section{In 2-D:}

$$
\begin{aligned}
& \mathbb{P}_{r}(x, y)=\operatorname{Span}\left\{x^{i} y^{j}, i, j \geq 0, i+j \leq r\right\} \\
& \widetilde{\mathbb{P}}_{r}(x, y)=\operatorname{Span}\left\{x^{i} y^{j}, i, j \geq 0, i+j=r\right\} \\
& \mathbb{Q}_{m, n}(x, y)=\operatorname{Span}\left\{x^{i} y^{j}, 0 \leq i \leq m, 0 \leq j \leq n\right\} \\
& \mathfrak{D}_{r}(x, y)=\left(\mathbb{P}_{r-1}(x, y)\right)^{2} \oplus \widetilde{\mathbb{P}}_{r-1}(x, y)\left[\begin{array}{l}
x \\
y
\end{array}\right]
\end{aligned}
$$

In 3-D:

$$
\begin{aligned}
& \mathbb{P}_{r}(x, y, z)=\operatorname{Span}\left\{x^{i} y^{j} z^{k}, i, j, k \geq 0, i+j+k \leq r\right\} \\
& \widetilde{\mathbb{P}}_{r}(x, y, z)=\operatorname{Span}\left\{x^{i} y^{j} z^{k}, i, j, k \geq 0, i+j+k=r\right\} \\
& \mathbb{Q}_{m, n, p}(x, y, z)=\operatorname{Span}\left\{x^{i} y^{j} z^{k}, 0 \leq i \leq m, 0 \leq j \leq n, 0 \leq k \leq p\right\} \\
& \mathbb{W}_{m, n}(x, y, z)=\operatorname{Span}\left\{x^{i} y^{j} z^{k}, i, j, k \geq 0, i+j \leq m, k \leq n\right\} \\
& \mathcal{S}_{r}(x, y, z)=\left\{u \in \widetilde{\mathbb{P}}_{r}^{3}, u_{1} x+u_{2} y+u_{3} z=0\right\} \\
& \mathcal{R}_{r}(x, y, z)=\mathbb{P}_{r-1}^{3} \oplus \mathcal{S}_{r} \\
& \mathfrak{D}_{r}(x, y, z)=\mathbb{P}_{r-1}^{3} \oplus \widetilde{\mathbb{P}}_{r-1}(x, y, z)\left[\begin{array}{l}
x \\
y \\
z
\end{array}\right]
\end{aligned}
$$

where $\mathcal{R}_{r}$ and $\mathfrak{D}_{r}$ are Nédélec's first family spaces for tetrahedra introduced by Nédélec (5), for $H($ curl) and $H($ div)formulation respectively, $\mathfrak{D}_{r}$ being also known as Raviart-Thomas finite elements (7). The space $\mathbb{W}_{r, r}$ is the finite element space used for continuous triangular prisms.

We also introduce the approximation space for continuous pyramidal elements (see (1))

$$
\mathbb{B}_{r}(x, y, z)=\mathbb{P}_{r}(x, y, z) \oplus \sum_{k=0}^{r-1} \mathbb{P}_{k}(x, y)\left(\frac{x y}{1-z}\right)^{r-k}
$$

which can be written on the cube $[-1,1] \times[0,1]$ as:

$$
\mathbb{C}_{r}(x, y, z)=\operatorname{Span}\left\{x^{i} y^{j}(1-z)^{k}, 0 \leq i, j \leq k \leq r\right\}
$$

using the transformation $T$ from the pyramid $\hat{K}(\hat{x}, \hat{y}, \hat{z})$ to the cube $[-1,1]^{2} \times[0,1]$ of coordinates $(\widetilde{x}, \widetilde{y}, \widetilde{z})$ :

$$
\left\{\begin{array}{l}
\hat{x}=\widetilde{x}(1-\widetilde{z}) \\
\hat{y}=\widetilde{y}(1-\widetilde{z}) \\
\hat{z}=\widetilde{z} .
\end{array}\right.
$$

The approximation space for $H($ curl $)$ pyramidal elements (see (2)) is

$$
\begin{aligned}
\mathcal{B}_{r}(x, y, z)= & \left.\mathbb{B}_{r-1}^{3}(x, y, z) \oplus\left\{\frac{x^{p} y^{p}}{(1-z)^{p+2}}\left[\begin{array}{c}
y(1-z) \\
\hat{x}(1-z) \\
x y
\end{array}\right], 0 \leq p \leq r-1\right\}\right\} \\
& \oplus\left\{\frac{x^{m} y^{n+2}}{(1-z)^{m+2}}\left[\begin{array}{c}
(1-z) \\
0 \\
x
\end{array}\right], \frac{x^{n+2} y^{m}}{(1-z)^{m+2}}\left[\begin{array}{c}
0 \\
(1-z) \\
y
\end{array}\right], 0 \leq m \leq n \leq r-2\right\} \\
& \oplus\left\{\frac{x^{p} y^{q}}{(1-z)^{p+q+1-r}}\left[\begin{array}{c}
(1-z) \\
0 \\
x
\end{array}\right], \frac{x^{q} y^{p}}{(1-z)^{p+q+1-r}}\left[\begin{array}{c}
0 \\
(1-z) \\
y
\end{array}\right], \begin{array}{l}
0 \leq p \leq r-1 \\
0 \leq q \leq r+1
\end{array}\right\} .
\end{aligned}
$$




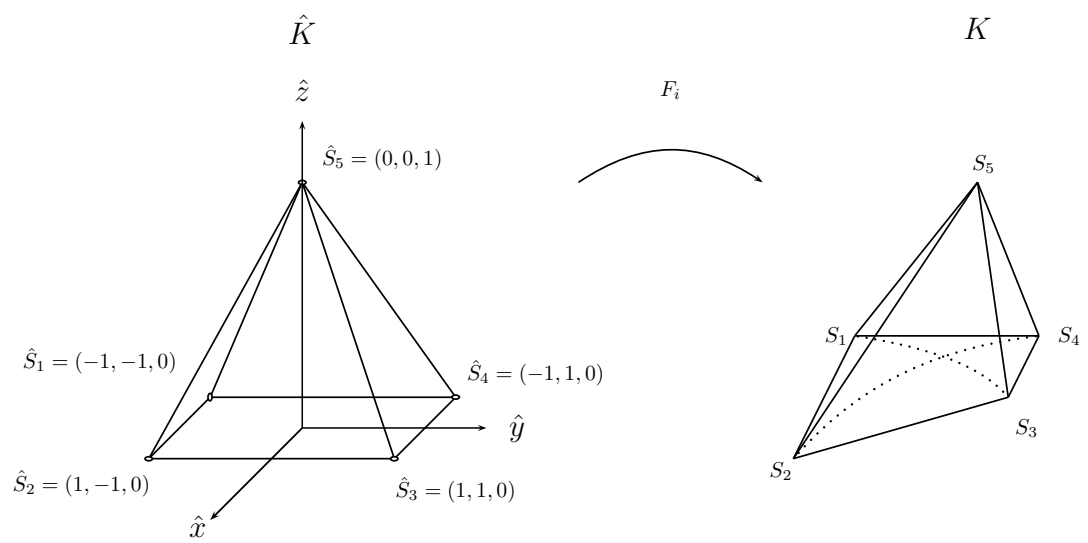

Figure 1: Transformation F for a pyramid

The finite element approximation is constructed by considering a transformation $F$ from a reference element $\hat{K}$ (unit tetrahedron, symmetric pyramid, unit prism and unit cube) to a real element $K$ on the mesh (see Fig. 1). This transformation is written as

$$
F=\sum_{1 \leq i \leq n_{i}} S_{i} \hat{\varphi}_{i}^{1}
$$

where $n_{i}$ is the number of vertices of the element, $S_{i}=\left(x_{i}, y_{i}, z_{i}\right)$ are the vertices and $\hat{\varphi}_{i}^{1}$ are mapping functions depending on the considered type of the element. The mapping functions are classical for tetrahedra, hexahedra and triangular prisms and can be found in (1) for pyramids.

By introducing some constants $A_{0}, A_{1}, A_{2}, A_{3}, C, C_{1}, C_{2}, C_{3}, D$ depending on the vertices $\left(S_{i}\right)$, the transformations $F$ writes as follows

Definition 2. The transformation $F$ for any element is written as:

- Tetrahedron: $F=A_{0}+A_{1} \hat{x}+A_{2} \hat{y}+A_{3} \hat{z}$

- Hexahedron: $F=A_{0}+A_{1} \hat{x}+A_{2} \hat{y}+A_{3} \hat{z}+C_{1} \hat{x} \hat{y}+C_{2} \hat{x} \hat{z}+C_{3} \hat{y} \hat{z}+D \hat{x} \hat{y} \hat{z}$

- Triangular prism: $F=A_{0}+A_{1} \hat{x}+A_{2} \hat{y}+A_{3} \hat{z}+C_{1} \hat{x} \hat{z}+C_{2} \hat{y} \hat{z}$

- Pyramid: $F=A_{0}+A_{1} \hat{x}+A_{2} \hat{y}+A_{3} \hat{z}+C \frac{\hat{x} \hat{y}}{(1-\hat{z})}$

There is a bijection between these constants and the vertices, that is the real element $K$ is uniquely determined by these constants (e.g. $A_{0}, A_{1}, A_{2}, A_{3}$ and $C$ for the pyramid).

Let us denote

$$
H(\operatorname{div}, \Omega)=\left\{u \in\left(L^{2}(\Omega)\right)^{3} \text { such that } \operatorname{div} u \in L^{2}(\Omega)\right\} .
$$

The unknown $u$ and test function $v$ of the variational formulation (2) belongs to the following finite element space:

$$
V_{h}=\left\{u \in H(\operatorname{div}, \Omega) \text { so that }\left.u\right|_{K} \in P_{r}^{F}\right\}
$$

where $P_{r}^{F}(K)$ denotes the finite element space on the element $K$ of the mesh. This space is built with the reference finite element space space $\hat{P}_{r}(\hat{K})$ thanks to Piola $H($ div $)$-conforming transform

$$
P_{r}^{F}(K)=\left\{u \text { so that }|D F| D F^{-1} u \circ F \in \hat{P}_{r}(\hat{K})\right\}
$$


where $D F$ the jacobian matrix of transformation $F$, and $|D F|$ its determinant. The space $\hat{P}_{r}(\hat{K})$ depends only on the reference element $\hat{K}$ and on the order of approximation $r$, and is thus independent of the element $K$. The approximate solution $u_{h}$ is the solution to the discrete variational formulation:

$$
\left\{\begin{array}{l}
\text { Find } u_{h} \in V_{h} \text { such that } \\
\forall v \in V_{h}, \quad-\omega^{2} \int_{\Omega} \frac{1}{\mu} u_{h} \cdot v_{h} d x+\int_{\Omega} \frac{1}{\rho} \operatorname{div} u_{h} \operatorname{div} v_{h} d x-i \omega \int_{\Sigma} u_{h} \cdot n v_{h} \cdot n d x=\int_{\Omega} f \cdot v_{h} d x
\end{array}\right.
$$

To obtain a convergence of $u_{h}$ towards the exact solution $u$ of the problem (1) in $O\left(h^{r}\right)$, where $h$ denotes the mesh size, a first necessary condition is to have the following inclusion (see (15)):

$$
P_{r}^{F} \supset \mathbb{P}_{r-1}(x, y, z)^{3} \text {. }
$$

Definition 3. The space $\hat{E}_{r}$ of order $r$ is the space of minimal dimension on $\hat{K}$ such that

$$
\hat{P}_{r}(\hat{K}) \supset \hat{E}_{r} \Leftrightarrow P_{r}^{F} \supset \mathbb{P}_{r-1}(x, y, z)^{3}
$$

for any element $K$.

Theorem 1. The spaces $\hat{E}_{r}$ are equal to:

\section{- Tetrahedron:}

$$
\hat{E}_{r}=\mathbb{P}_{r-1}(\hat{x}, \hat{y}, \hat{z})^{3}
$$

\section{- Hexahedron:}

$$
\begin{aligned}
\hat{E}_{1}=\mathbb{P}_{1}(\hat{x}, \hat{y}, \hat{z})^{3} & \oplus\left[\begin{array}{c}
\hat{x} \hat{y} \\
0 \\
-\hat{y} \hat{z}
\end{array}\right] \oplus\left[\begin{array}{c}
\hat{x} \hat{z} \\
-\hat{y} \hat{z} \\
0
\end{array}\right] \oplus\left[\begin{array}{c}
0 \\
-\hat{x} \hat{y} \\
\hat{x} \hat{z}
\end{array}\right] \\
& \oplus\left[\begin{array}{c}
\hat{x}^{2} \hat{z} \\
0 \\
-\hat{x} \hat{z}^{2}
\end{array}\right] \oplus\left[\begin{array}{c}
\hat{x}^{2} \hat{y} \\
-\hat{x} \hat{y}^{2} \\
0
\end{array}\right] \oplus\left[\begin{array}{c}
0 \\
-\hat{y}^{2} \hat{z} \\
\hat{y} \hat{z}^{2}
\end{array}\right] \\
& \oplus\left[\begin{array}{c}
\hat{x}^{2} \\
-\hat{x} \hat{y} \\
-\hat{x} \hat{z}
\end{array}\right] \oplus\left[\begin{array}{c}
-\hat{x} \hat{y} \\
\hat{y}^{2} \\
-\hat{y} \hat{z}
\end{array}\right] \oplus\left[\begin{array}{c}
-\hat{x} \hat{z} \\
-\hat{y} \hat{z} \\
\hat{z}^{2}
\end{array}\right]
\end{aligned}
$$

For $r>1$

$$
\begin{aligned}
\hat{E}_{r}= & \mathbb{Q}_{r+1, r-1, r-1}(\hat{x}, \hat{y}, \hat{z}) \times \mathbb{Q}_{r-1, r+1, r-1}(\hat{x}, \hat{y}, \hat{z}) \times \mathbb{Q}_{r-1, r-1, r+1}(\hat{x}, \hat{y}, \hat{z}) \\
& \left.\oplus\left\{\begin{array}{c}
\hat{x}^{i} \hat{y}^{r} \hat{z}^{j} \\
0 \\
0
\end{array}\right] \oplus\left[\begin{array}{c}
0 \\
\hat{x}^{j} \hat{y}^{i} \hat{z}^{r} \\
0
\end{array}\right] \oplus\left[\begin{array}{c}
0 \\
0 \\
\hat{x}^{r} \hat{y}^{j} \hat{z}^{i}
\end{array}\right] \begin{array}{c}
0 \leq i \leq r \\
0 \leq j \leq r-1
\end{array}\right\} \\
& \left.\oplus\left\{\begin{array}{c}
\hat{x}^{i} \hat{y}^{j} \hat{z}^{r} \\
0 \\
0
\end{array}\right] \oplus\left[\begin{array}{c}
0 \\
\hat{x}^{r} \hat{y}^{i} \hat{z}^{j} \\
0
\end{array}\right] \oplus\left[\begin{array}{c}
0 \\
0 \\
\hat{x}^{j} \hat{y}^{r} \hat{z}^{i}
\end{array}\right] \begin{array}{c}
0 \leq i \leq r \\
0 \leq j \leq r-1
\end{array}\right\} \\
& \left.\oplus\left\{\begin{array}{c}
\hat{x}^{r+1} \hat{y}^{j} \hat{z}^{r} \\
0 \\
-\hat{x}^{r} \hat{y}^{j} \hat{z}^{r+1}
\end{array}\right] \oplus\left[\begin{array}{c}
0 \\
\hat{x}^{j} \hat{y}^{r+1} \hat{z}^{r} \\
-\hat{x}^{j} \hat{y}^{r} \hat{z}^{r+1}
\end{array}\right] \oplus\left[\begin{array}{c}
\hat{x}^{r+1} \hat{y}^{r} \hat{z}^{j} \\
-\hat{x}^{r} \hat{y}^{r+1} \hat{z}^{j} \\
0
\end{array}\right] \quad \begin{array}{c}
0 \leq j \leq r-1 \\
0
\end{array}\right\}
\end{aligned}
$$

\section{- Triangular prism:}

$\hat{E}_{r}=\left(\mathbb{W}_{r-1, r}(\hat{x}, \hat{y}, \hat{z}) \oplus \widetilde{\mathbb{P}}_{r}(\hat{x}, \hat{y}) \mathbb{P}_{r-1}(\hat{z})\right)^{2} \otimes\left(\mathbb{W}_{r-2, r+1}(\hat{x}, \hat{y}, \hat{z}) \oplus \widetilde{\mathbb{P}}_{r-1}(\hat{x}, \hat{y}) \mathbb{P}_{r}(\hat{z})\right) \oplus \widetilde{\mathbb{P}}_{r-1}(\hat{x}, \hat{y}) \hat{z}^{r}\left[\begin{array}{c}-\hat{x} \\ -\hat{y} \\ \hat{z}\end{array}\right]$ 


\section{- Pyramid:}

$$
\left.\begin{array}{rl}
\hat{E}_{r}=\mathbb{B}_{r-1}(\hat{x}, \hat{y}, \hat{z})^{3} & \oplus\left\{\left[\begin{array}{c}
\frac{\hat{x}^{n+1} \hat{y}^{m}}{(1-\hat{z})^{m+1}} \\
0 \\
0
\end{array}\right] \oplus\left[\begin{array}{c}
0 \\
\frac{\hat{x}^{m} \hat{y}^{n+1}}{(1-\hat{z})^{m+1}} \\
0
\end{array}\right] 0 \leq m \leq n \leq r-1\right\} \\
& \oplus\left\{\left[\begin{array}{c}
\frac{\hat{x}^{m+1} \hat{y}^{n+1}}{(1-\hat{z})^{m+2}} \\
0 \\
-\frac{\hat{x}^{m} \hat{y}^{n+1}}{(1-\hat{z})^{m+1}}
\end{array}\right] \oplus\left[\begin{array}{c}
0 \\
\frac{\hat{x}^{n+1} \hat{y}^{m+1}}{(1-\hat{z})^{m+2}} \\
-\frac{\hat{x}^{n+1} \hat{y}^{m}}{(1-\hat{z})^{m+1}}
\end{array}\right] 0 \leq m \leq n \leq r-1\right.
\end{array}\right\}
$$

Proof. The proof will be completed for the pyramid and the hexahedron. Let us begin with the pyramid, for which the transformation $F$ is equal to

$$
F=A_{0}+A_{1} \hat{x}+A_{2} \hat{y}+A_{3} \hat{z}+C \frac{\hat{x} \hat{y}}{(1-\hat{z})}
$$

where $(\hat{x}, \hat{y}, \hat{z})$ are the coordinates on the symmetric pyramid. We will express each function either in $(\hat{x}, \hat{y}, \hat{z})$ coordinates, or either in $(\widetilde{x}, \widetilde{y}, \widetilde{z})$ coordinates of the cube $[-1,1]^{2} \times[0,1]$ defined by the relations

$$
\left\{\begin{array}{l}
\hat{x}=\widetilde{x}(1-\widetilde{z}) \\
\hat{y}=\widetilde{y}(1-\widetilde{z}) \\
\hat{z}=\widetilde{z} .
\end{array}\right.
$$

The derivatives of $F$ with respect to coordinates of $\hat{K}$ are equal to:

$$
\begin{aligned}
& \frac{\partial F}{\partial \hat{x}}=A_{1}+C \frac{\hat{y}}{(1-\hat{z})}=A_{1}+C \widetilde{y} \\
& \frac{\partial F}{\partial \hat{y}}=A_{2}+C \frac{\hat{x}}{(1-\hat{z})}=A_{2}+C \widetilde{x} \\
& \frac{\partial F}{\partial \hat{z}}=A_{3}+C \frac{\hat{x} \hat{y}}{(1-\hat{z})^{2}}=A_{3}+C \widetilde{x} \widetilde{y} .
\end{aligned}
$$

The columns of the comatrix of DF are equal to:

$$
\begin{aligned}
& \operatorname{comat}(D F)_{x}=\frac{\partial F}{\partial \hat{y}} \times \frac{\partial F}{\partial \hat{z}}=A_{2} \times A_{3}+C \times A_{3} \widetilde{x}+A_{2} \times C \widetilde{x} \widetilde{y} \\
& \operatorname{comat}(D F)_{y}=\frac{\partial F}{\partial \hat{z}} \times \frac{\partial F}{\partial \hat{x}}=A_{3} \times A_{1}+A_{3} \times C \widetilde{y}+C \times A_{1} \widetilde{x} \widetilde{y} \\
& \operatorname{comat}(D F)_{z}=\frac{\partial F}{\partial \hat{x}} \times \frac{\partial F}{\partial \hat{y}}=A_{1} \times A_{2}+A_{1} \times C \widetilde{x}+C \times A_{2} \widetilde{y} .
\end{aligned}
$$

Let us take a polynomial $\mathrm{p}$ in $\mathbb{P}_{r-1}^{3}$. Piola transform writes:

$$
\hat{p}=|D F| D F^{-1} p \circ F
$$

where $|D F|$ is the determinant of the jacobian matrix. By using comatrix, the relation is simpler:

$$
\hat{p}=\operatorname{comat}(D F)^{*} p \circ F .
$$

Because of the optimality of $\mathbb{C}_{r}$ (see (1)), considering a polynomial of $\mathbb{P}_{r-1}^{3}$ on the pyramid $\hat{K}$ is equivalent to consider a polynomial of $\mathbb{C}_{r-1}^{3}$ on the unit cube $[-1,1]^{2} \times[0,1]$, that is:

$$
p \circ F=\widetilde{x}^{i} \widetilde{y}^{j}(1-\widetilde{z})^{k} U, \quad i, j \leq k \leq r-1
$$


with $U$ a constant vector in $\mathbb{R}^{3}$. Therefore $\hat{p}$ is equal to:

$$
\begin{aligned}
\hat{p}= & \operatorname{comat}(D F)^{*} \widetilde{x}^{i} \widetilde{y}^{j}(1-\widetilde{z})^{k} U=\widetilde{x}^{i} \widetilde{y}^{j}(1-\widetilde{z})^{k}\left[\begin{array}{c}
\left(A_{2}, A_{3}, U\right) \\
\left(A_{3}, A_{1}, U\right) \\
\left(A_{1}, A_{2}, U\right)
\end{array}\right] \\
& +\widetilde{x}^{i} \widetilde{y}^{j}(1-\widetilde{z})^{k}\left(\left(C, A_{3}, U\right)\left[\begin{array}{c}
\widetilde{x} \\
-\widetilde{y} \\
0
\end{array}\right]+\left(A_{2}, C, U\right)\left[\begin{array}{c}
\widetilde{x} \widetilde{y} \\
0 \\
-\widetilde{y}
\end{array}\right]+\left(C, A_{1}, U\right)\left[\begin{array}{c}
0 \\
\widetilde{x} \widetilde{y} \\
-\widetilde{x}
\end{array}\right]\right)
\end{aligned}
$$

where $(A, B, C)$ denotes the determinant of the $3 \times 3$ matrix whose columns are $A, B$ and $C$. By varying values of $A_{1}, A_{2}, A_{3}$ and $U$, the first term generates all monomials of $\mathbb{C}_{r-1}^{3}$. For the other terms, we distinguish four cases

1. If $i, j<k$, all the terms belongs to $\mathbb{C}_{r-1}^{3}$.

2. If $i=k, j<k$, by removing components in $\mathbb{C}_{r-1}^{3}$, it remains

$$
\left[\begin{array}{c}
\widetilde{x}^{k+1} \widetilde{y}^{m}(1-\widetilde{z})^{k} \\
0 \\
0
\end{array}\right], \quad \widetilde{x}^{k} \widetilde{y}^{j}(1-\widetilde{z})^{k}\left[\begin{array}{c}
0 \\
\widetilde{x} \widetilde{y} \\
-\widetilde{x}
\end{array}\right], \quad m \leq k, j<k .
$$

3. If $j=k, i<k$, by removing components in $\mathbb{C}_{r-1}^{3}$, it remains

$$
\left[\begin{array}{c}
0 \\
\widetilde{x}^{m} \widetilde{y}^{k+1}(1-\widetilde{z})^{k} \\
0
\end{array}\right], \quad \widetilde{x}^{j} \widetilde{y}^{k}(1-\widetilde{z})^{k}\left[\begin{array}{c}
\widetilde{x} \widetilde{y} \\
0 \\
-\widetilde{y}
\end{array}\right], \quad m \leq k, j<k .
$$

4. If $i=k, j=k$, we have the following terms

$$
\left[\begin{array}{c}
\widetilde{x}^{k+1} \widetilde{y}^{k}(1-\widetilde{z})^{k} \\
0 \\
0
\end{array}\right]-\left[\begin{array}{c}
0 \\
\widetilde{x}^{k} \widetilde{y}^{k+1}(1-\widetilde{z})^{k} \\
0
\end{array}\right], \quad \widetilde{x}^{k} \widetilde{y}^{k}(1-\widetilde{z})^{k}\left[\begin{array}{c}
\widetilde{x} \widetilde{y} \\
0 \\
-\widetilde{y}
\end{array}\right], \quad \widetilde{x}^{k} \widetilde{y}^{k}(1-\widetilde{z})^{k}\left[\begin{array}{c}
0 \\
\widetilde{x} \widetilde{y} \\
-\widetilde{x}
\end{array}\right] .
$$

The two first terms are already treated in the second and third case and the last two terms can be added to the second and third case by extending $j$ until $k$ (instead of $j<k$ ).

Finally, by taking polynomials $p$ in $\mathbb{P}_{r-1}^{3}$, the following space is generated:

$$
\begin{aligned}
& \mathbb{C}_{r-1}^{3} \oplus\left\{\left[\begin{array}{c}
\widetilde{x}^{k+1} \widetilde{y}^{m}(1-\widetilde{z})^{k} \\
0 \\
0
\end{array}\right], \quad\left[\begin{array}{c}
\widetilde{x}^{m} \widetilde{y}^{k+1}(1-\widetilde{z})^{k} \\
0
\end{array}\right], \quad 0 \leq m \leq k \leq r-1\right\} \\
& \oplus\left\{\left[\begin{array}{c}
\widetilde{x}^{j+1} \widetilde{y}^{k+1}(1-\widetilde{z})^{k} \\
0 \\
-\widetilde{x}^{j} \widetilde{y}^{k+1}(1-\widetilde{z})^{k}
\end{array}\right], \quad\left[\begin{array}{c}
0 \\
\widetilde{x}^{k+1} \widetilde{y}^{j+1}(1-\widetilde{z})^{k} \\
-\widetilde{x}^{k+1} \widetilde{y}^{j}(1-\widetilde{z})^{k}
\end{array}\right], \quad 0 \leq j \leq k \leq r-1\right\} .
\end{aligned}
$$

When we write this space in coordinates $(\hat{x}, \hat{y}, \hat{z})$ we recover the claimed space $\hat{E}_{r}$ for the pyramid.

We now consider the following transformation for a general hexahedron:

$$
F=A_{0}+A_{1} \hat{x}+A_{2} \hat{y}+A_{3} \hat{z}+C_{1} \hat{x} \hat{y}+C_{2} \hat{x} \hat{z}+C_{3} \hat{y} \hat{z}+D \hat{x} \hat{y} \hat{z} .
$$

The derivatives of $\mathrm{F}$ are equal to:

$$
\begin{aligned}
& \frac{\partial F}{\partial \hat{x}}=A_{1}+C_{1} \hat{y}+C_{2} \hat{z}+D \hat{y} \hat{z} \\
& \frac{\partial F}{\partial \hat{y}}=A_{2}+C_{1} \hat{x}+C_{3} \hat{z}+D \hat{x} \hat{z} \\
& \frac{\partial F}{\partial \hat{z}}=A_{3}+C_{2} \hat{x}+C_{3} \hat{y}+D \hat{x} \hat{y} .
\end{aligned}
$$


The columns of the comatrix of DF are equal to:

$$
\begin{aligned}
\operatorname{comat}(D F)_{x}= & \frac{\partial F}{\partial \hat{y}} \times \frac{\partial F}{\partial \hat{z}}=A_{2} \times A_{3}+\hat{x}\left(C_{1} \times A_{3}+A_{2} \times C_{2}\right)+\hat{y}\left(A_{2} \times C_{3}\right)+\hat{z}\left(C_{3} \times A_{3}\right) \\
& +\hat{x} \hat{y}\left(A_{2} \times D+C_{1} \times C_{3}\right)+\hat{x} \hat{z}\left(D \times A_{3}+C_{3} \times C_{2}\right)+\hat{x}^{2}\left(C_{1} \times C_{2}\right) \\
& +\hat{x}^{2} \hat{y}\left(C_{1} \times D\right)+\hat{x}^{2} \hat{z}\left(D \times C_{2}\right) \\
\operatorname{comat}(D F)_{y}= & \frac{\partial F}{\partial \hat{z}} \times \frac{\partial F}{\partial \hat{x}}=A_{3} \times A_{1}+\hat{x}\left(C_{2} \times A_{1}\right)+\hat{y}\left(A_{3} \times C_{1}+C_{3} \times A_{1}\right)+\hat{z}\left(A_{3} \times C_{2}\right) \\
& +\hat{x} \hat{y}\left(D \times A_{1}+C_{2} \times C_{1}\right)+\hat{y} \hat{z}\left(A_{3} \times D+C_{3} \times C_{2}\right)+\hat{y}^{2}\left(C_{3} \times C_{1}\right) \\
& +\hat{y}^{2} \hat{x}\left(D \times C_{1}\right)+\hat{y}^{2} \hat{z}\left(C_{3} \times D\right) \\
& \frac{\partial F}{\partial \hat{x}} \times \frac{\partial F}{\partial \hat{y}}=A_{1} \times A_{2}+\hat{x}\left(A_{1} \times C_{1}\right)+\hat{y}\left(C_{1} \times A_{2}\right)+\hat{z}\left(A_{1} \times C_{3}+C_{2} \times A_{2}\right) \\
& +\hat{x} \hat{z}\left(C_{2} \times C_{1}+A_{1} \times D\right)+\hat{y} \hat{z}\left(C_{1} \times C_{3}+D \times A_{2}\right)+\hat{z}^{2}\left(C_{2} \times C_{3}\right) \\
& +\hat{x} \hat{z}^{2}\left(C_{2} \times D\right)+\hat{y} \hat{z}^{2}\left(D \times C_{3}\right) .
\end{aligned}
$$

Let us begin with a polynomial $\mathrm{p}$ in $\mathbb{P}_{r-1}^{3}$. Because of the optimality of $\mathbb{Q}_{r}$ (see (1)), it is equivalent to consider a polynomial of $\mathbb{Q}_{r-1}^{3}$ :

$$
p \circ F=\hat{x}^{i} \hat{y}^{j} \hat{z}^{k} U, \quad i, j \leq k \leq r-1
$$

with $U$ a constant vector in $\mathbb{R}^{3}$. Therefore $\hat{p}$ is equal to:

$$
\begin{aligned}
\hat{p}= & \operatorname{comat}(D F)^{*} \hat{x}^{i} \hat{y}^{j} \hat{z}^{k} U=\hat{x}^{i} \hat{y}^{j} \hat{z}^{k}\left\{\left[\begin{array}{c}
\left(A_{2}, A_{3}, U\right) \\
\left(A_{3}, A_{1}, U\right) \\
\left(A_{1}, A_{2}, U\right)
\end{array}\right]+\left(C_{1}, A_{3}, U\right)\left[\begin{array}{c}
\hat{x} \\
-\hat{y} \\
0
\end{array}\right]\right. \\
& +\left(A_{2}, C_{2}, U\right)\left[\begin{array}{c}
\hat{x} \\
0 \\
-\hat{z}
\end{array}\right]+\left(A_{2}, C_{3}, U\right)\left[\begin{array}{l}
\hat{y} \\
0 \\
0
\end{array}\right]+\left(C_{3}, A_{3}, U\right)\left[\begin{array}{c}
\hat{z} \\
0 \\
0
\end{array}\right]+\left(A_{2}, D, U\right)\left[\begin{array}{c}
\hat{x} \hat{y} \\
0 \\
-\hat{y} \hat{z}
\end{array}\right] \\
& +\left(C_{3}, C_{1}, U\right)\left[\begin{array}{c}
-\hat{x} \hat{y} \\
\hat{y}^{2} \\
-\hat{y} \hat{z}
\end{array}\right]+\left(D, A_{3}, U\right)\left[\begin{array}{c}
\hat{x} \hat{z} \\
-\hat{y} \hat{z} \\
0
\end{array}\right]+\left(C_{1}, C_{2}, U\right)\left[\begin{array}{c}
\hat{x}^{2} \\
-\hat{x} \hat{y} \\
-\hat{x} \hat{z}
\end{array}\right] \\
& +\left(C_{1}, D, U\right)\left[\begin{array}{c}
\hat{x}^{2} \hat{y} \\
-\hat{x} \hat{y}^{2} \\
0
\end{array}\right]+\left(D, C_{2}, U\right)\left[\begin{array}{c}
\hat{x} \hat{z}^{2} \hat{z} \\
0 \\
-\hat{x} \hat{z}^{2}
\end{array}\right]+\left(C_{2}, A_{1}, U\right)\left[\begin{array}{c}
0 \\
\hat{x} \\
0
\end{array}\right]+\left(C_{3}, A_{1}, U\right)\left[\begin{array}{c}
0 \\
\hat{y} \\
-\hat{z}
\end{array}\right] \\
& +\left(A_{3}, C_{2}, U\right)\left[\begin{array}{c}
0 \\
\hat{z} \\
0
\end{array}\right]+\left(D, A_{1}, U\right)\left[\begin{array}{c}
\hat{x} \hat{y} \\
-\hat{x} \hat{z}
\end{array}\right]+\left(C_{3}, C_{2}, U\right)\left[\begin{array}{c}
\hat{x} \hat{z} \\
\hat{y} \hat{z} \\
-\hat{z}^{2}
\end{array}\right] \\
& \left.+\left(C_{3}, D, U\right)\left[\begin{array}{c}
0 \\
\hat{y}^{2} \hat{z} \\
-\hat{y} \hat{z}^{2}
\end{array}\right]+\left(C_{1}, A_{2}, U\right)\left[\begin{array}{l}
0 \\
0 \\
\hat{y}
\end{array}\right]+\left(A_{1}, C_{1}, U\right)\left[\begin{array}{c}
0 \\
0 \\
\hat{x}
\end{array}\right]\right\} .
\end{aligned}
$$

The coefficients $\left(A_{2}, A_{3}, U\right),\left(A_{3}, A_{1}, U\right),\left(A_{1}, A_{2}, U\right),\left(C_{1}, A_{3}, U\right),\left(A_{2}, C_{2}, U\right),\left(A_{2}, C_{3}, U\right),\left(C_{3}, A_{3}, U\right),\left(A_{2}, D, U\right)$, $\left(C_{3}, C_{1}, U\right),\left(D, A_{3}, U\right),\left(C_{1}, C_{2}, U\right),\left(C_{1}, D, U\right),\left(D, C_{2}, U\right),\left(C_{2}, A_{1}, U\right),\left(C_{3}, A_{1}, U\right),\left(A_{3}, C_{2}, U\right),\left(D, A_{1}, U\right)$, $\left(C_{3}, C_{2}, U\right),\left(C_{3}, D, U\right),\left(C_{1}, A_{2}, U\right),\left(A_{1}, C_{1}, U\right)$ viewed as functions of variables $U, A_{0}, A_{1}, A_{2}, A_{3}, C_{1}, C_{2}, C_{3}, D$ are linearly independent, therefore all the associated monomials are needed to obtain $\mathbb{P}_{r-1}^{3}$. By a simple recombination 
of these monomials, they can be enumerated as follows:

$$
\begin{aligned}
& {\left[\begin{array}{l}
1 \\
0 \\
0
\end{array}\right],\left[\begin{array}{l}
0 \\
1 \\
0
\end{array}\right],\left[\begin{array}{l}
0 \\
0 \\
1
\end{array}\right],\left[\begin{array}{l}
\hat{x} \\
0 \\
0
\end{array}\right],\left[\begin{array}{l}
0 \\
\hat{x} \\
0
\end{array}\right],\left[\begin{array}{l}
0 \\
0 \\
\hat{x}
\end{array}\right],\left[\begin{array}{l}
\hat{y} \\
0 \\
0
\end{array}\right],\left[\begin{array}{l}
0 \\
\hat{y} \\
0
\end{array}\right],\left[\begin{array}{c}
0 \\
0 \\
\hat{y}
\end{array}\right]} \\
& {\left[\begin{array}{l}
\hat{z} \\
0 \\
0
\end{array}\right],\left[\begin{array}{l}
0 \\
\hat{z} \\
0
\end{array}\right],\left[\begin{array}{l}
0 \\
0 \\
\hat{z}
\end{array}\right],\left[\begin{array}{c}
\hat{x} \hat{y} \\
0 \\
-\hat{y} \hat{z}
\end{array}\right],\left[\begin{array}{c}
\hat{x} \hat{z} \\
-\hat{y} \hat{z} \\
0
\end{array}\right],\left[\begin{array}{c}
0 \\
\hat{x} \hat{y} \\
-\hat{x} \hat{z}
\end{array}\right]} \\
& {\left[\begin{array}{c}
\hat{x}^{2} \\
-\hat{x} \hat{y} \\
-\hat{x} \hat{z}
\end{array}\right],\left[\begin{array}{c}
-\hat{x} \hat{y} \\
\hat{y}^{2} \\
-\hat{y} \hat{z}
\end{array}\right],\left[\begin{array}{c}
-\hat{x} \hat{z} \\
-\hat{y} \hat{z} \\
\hat{z}^{2}
\end{array}\right],\left[\begin{array}{c}
\hat{x}^{2} \hat{y} \\
-\hat{x} \hat{y}^{2} \\
0
\end{array}\right],\left[\begin{array}{c}
\hat{x}^{2} \hat{z} \\
0 \\
-\hat{x} \hat{z}^{2}
\end{array}\right],\left[\begin{array}{c}
0 \\
\hat{y}^{2} \hat{z} \\
-\hat{y} \hat{z}^{2}
\end{array}\right] .}
\end{aligned}
$$

These monomials have to be multiplied by $\hat{x}^{i} \hat{y}^{j} \hat{z}^{k}$ with $i, j, k \leq r-1$ to obtain the needed functions to generate $\mathbb{P}_{r-1}^{3}$. When $r=1$, all these monomials are needed, and no further manipulation is necessary. When $r>1$, we will make manipulations in order to obtain a suitable expression of $\hat{E}_{r}$.

Because of the presence of $(\hat{x}, 0,0)$, it is immediate that the following functions:

$$
\left\{\left[\begin{array}{c}
\hat{x}^{r} \hat{y}^{i} \hat{z}^{j} \\
0 \\
0
\end{array}\right], \quad 0 \leq i, j \leq r-1\right\}
$$

belong to $\hat{E}_{r}$. Let us now consider the following combination between functions of $\hat{E}_{r}$ :

$$
\left[\begin{array}{c}
\hat{x}^{i+2} \hat{y}^{j} \hat{z}^{k} \\
-\hat{x}^{i+1} \hat{y}^{j+1} \hat{z}^{k} \\
-\hat{x}^{i+1} \hat{y}^{j} \hat{z}^{k+1}
\end{array}\right]+\left[\begin{array}{c}
0 \\
\hat{x}^{i^{\prime}+1} \hat{y}^{j^{\prime}} \hat{z}^{k^{\prime}} \\
0
\end{array}\right]+\left[\begin{array}{c}
0 \\
0 \\
\hat{x}^{\ell+1} \hat{y}^{m} \hat{z}^{n}
\end{array}\right]=\left[\begin{array}{c}
\hat{x}^{i+2} \hat{y}^{j} \hat{z}^{k} \\
0 \\
0
\end{array}\right]
$$

All the functions of this equality belong to $\hat{E}_{r}$ if

$$
0 \leq i, j, k, i^{\prime}, j^{\prime}, k^{\prime}, \ell, m, n \leq r-1
$$

This equality becomes true if we have

$$
\mid \begin{array}{ll}
i^{\prime}=i, & j^{\prime}=j+1, \quad k^{\prime}=k \\
\ell=i, & m=j, \quad n=k+1
\end{array}
$$

This choice of indices is possible if $j, k \leq r-2$. As a result, the monomials $\left(\hat{x}^{i+2} \hat{y}^{j} \hat{z}^{k}, 0,0\right)$ belong to $\hat{E}_{r}$ if $i \leq$ $r-1, j, k \leq r-2$. The following combination is now considered:

$$
-\left[\begin{array}{c}
\hat{x}^{i+2} \hat{y}^{j} \hat{z}^{k} \\
-\hat{x}^{i+1} \hat{y}^{j+1} \hat{z}^{k} \\
-\hat{x}^{i+1} \hat{y}^{j} \hat{z}^{k+1}
\end{array}\right]+2\left[\begin{array}{c}
\hat{x}^{i^{\prime}+2} \hat{y}^{j^{\prime}} \hat{z}^{k^{\prime}+1} \\
0 \\
-\hat{x}^{i^{\prime}+1} \hat{y}^{j^{\prime}} \hat{z}^{k^{\prime}+2}
\end{array}\right]-\left[\begin{array}{c}
0 \\
\hat{x}^{\ell+1} \hat{y}^{m+1} \hat{z}^{n} \\
-\hat{x}^{\ell+1} \hat{y}^{m} \hat{z}^{n+1}
\end{array}\right]=\left[\begin{array}{c}
\hat{x}^{i+2} \hat{y}^{j} \hat{z}^{k} \\
0 \\
0
\end{array}\right]
$$

This identity is true as soon as:

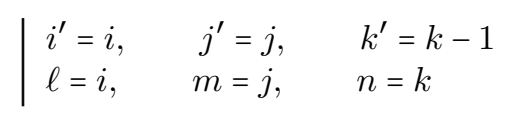

This choice of indices is possible if $k \geq 1$. Therefore $\left(\hat{x}^{i+2} \hat{y}^{j} \hat{z}^{k}, 0,0\right)$ belongs to $\hat{E}_{r}$ if $1 \leq k \leq r-1$ and $i, j \leq r-1$. With a similar combination we can also find $\left(\hat{x}^{i+2} \hat{y}^{j} \hat{z}^{k}, 0,0\right)$ in $\hat{E}_{r}$ if $1 \leq j \leq r-1$ and $i, k \leq r-1$.

As a result, if $r>1$, the following monomials belongs to $\hat{E}_{r}$ :

$$
\left\{\left[\begin{array}{c}
\hat{x}^{r+1} \hat{y}^{i} \hat{z}^{j} \\
0 \\
0
\end{array}\right], \quad 0 \leq i, j \leq r-1\right\}
$$


Let us now consider the following combination:

$$
-\left[\begin{array}{c}
-\hat{x}^{i+1} \hat{y}^{j+1} \hat{z}^{k} \\
\hat{x}^{i} \hat{y}^{j+2} \hat{z}^{k} \\
-\hat{x}^{i} \hat{y}^{j+1} \hat{z}^{k+1}
\end{array}\right]+\left[\begin{array}{c}
\hat{x}^{i^{\prime}+1} \hat{y}^{j^{\prime}+1} \hat{z}^{k^{\prime}} \\
0 \\
-\hat{x}^{i^{\prime}} \hat{y}^{j^{\prime}+1} \hat{z}^{k^{\prime}+1}
\end{array}\right]-\left[\begin{array}{c}
\hat{x}^{\ell+2} \hat{y}^{m+1} \hat{z}^{n} \\
-\hat{x}^{\ell+1} \hat{y}^{m+2} \hat{z}^{n} \\
0
\end{array}\right]=\left[\begin{array}{c}
\hat{x}^{i+1} \hat{y}^{j+1} \hat{z}^{k} \\
0 \\
0
\end{array}\right]
$$

This identity becomes true when

$$
\mid \begin{aligned}
& i^{\prime}=i, \quad j^{\prime}=j, \quad k^{\prime}=k \\
& \ell=i-1, \quad m=j, \quad n=k
\end{aligned}
$$

This choice of indices is possible if $i \geq 1$. If $r>1$ and by taking $i=r-1$, we obtain the function $\left(\hat{x}^{r} \hat{y}^{r} \hat{z}^{k}, 0,0\right)$ in $\hat{E}_{r}$ with $k \leq r-1$. Similarly, we can obtain the function $\left(\hat{x}^{r} \hat{y}^{k} \hat{z}^{r}, 0,0\right)$. Because of the presence of the monomials $(\hat{y}, 0,0)$ and $(\hat{z}, 0,0)$, the functions $\left(\hat{x}^{i} \hat{y}^{r} \hat{z}^{k}, 0,0\right)$ and $\left(\hat{x}^{i} \hat{y}^{r} \hat{z}^{k}, 0,0\right)$ with $i, k \leq r-1$ belong to $\hat{E}_{r}$. As a result, the following monomials are included in $\hat{E}_{r}$ :

$$
\left\{\left[\begin{array}{c}
\hat{x}^{i} \hat{y}^{r} \hat{z}^{j} \\
0 \\
0
\end{array}\right],\left[\begin{array}{c}
\hat{x}^{i} \hat{y}^{j} \hat{z}^{r} \\
0 \\
0
\end{array}\right], \quad 0 \leq i \leq r, \quad 0 \leq j \leq r-1\right\}
$$

By regrouping the different results, and exploiting symmetries, we have proved that $\hat{E}_{r}$ contains the following functions:

$$
\left\{\begin{array}{c}
{\left[\begin{array}{c}
\hat{x}^{i} \hat{y}^{j} \hat{z}^{k} \\
0 \\
0
\end{array}\right],\left[\begin{array}{c}
0 \\
\hat{x}^{j} \hat{y}^{i} \hat{z}^{k} \\
0
\end{array}\right],\left[\begin{array}{c}
0 \\
0 \\
\hat{x}^{j} \hat{y}^{k} \hat{z}^{i}
\end{array}\right], \quad i \leq r+1, \quad j, k \leq r-1} \\
{\left[\begin{array}{c}
\hat{x}^{i} \hat{y}^{r} \hat{z}^{k} \\
0 \\
0
\end{array}\right],\left[\begin{array}{c}
\hat{x}^{i} \hat{y}^{k} \hat{z}^{r} \\
0 \\
0
\end{array}\right],\left[\begin{array}{c}
0 \\
\hat{x}^{r} \hat{y}^{i} \hat{z}^{k} \\
0
\end{array}\right],\left[\begin{array}{c}
0 \\
\hat{x}^{k} \hat{y}^{i} \hat{z}^{r} \\
0
\end{array}\right],\left[\begin{array}{c}
0 \\
0 \\
\hat{x}^{r} \hat{y}^{k} \hat{z}^{i}
\end{array}\right],\left[\begin{array}{c}
0 \\
0 \\
\hat{x}^{k} \hat{y}^{r} \hat{z}^{i}
\end{array}\right], \quad i \leq r, \quad k \leq r-1}
\end{array}\right\}
$$

Let us now consider the monomials

$$
\left[\begin{array}{c}
\hat{x}^{i+2} \hat{y}^{j+1} \hat{z}^{k} \\
-\hat{x}^{i+1} \hat{y}^{j+2} \hat{z}^{k} \\
0
\end{array}\right],\left[\begin{array}{c}
\hat{x}^{i+2} \hat{y}^{j} \hat{z}^{k+1} \\
0 \\
-\hat{x}^{i+1} \hat{y}^{j} \hat{z}^{k+2}
\end{array}\right],\left[\begin{array}{c}
0 \\
\hat{x}^{i} \hat{y}^{j+2} \hat{z}^{k} \\
-\hat{x}^{i} \hat{y}^{j+1} \hat{z}^{k+1}
\end{array}\right]
$$

These functions can be written as combination of the previous functions that are included in $\hat{E}_{r}$, except when

$$
\begin{aligned}
& i=j=r-1 \text { for the first monomial } \\
& i=k=r-1 \text { for the second monomial } \\
& j=k=r-1 \text { for the third monomial }
\end{aligned}
$$

With this choice of indices, we have found the last monomials of $\hat{E}_{r}$.

Following the principles used to construct $H(c u r l)$ optimal spaces defined in (2), the optimal space on $\hat{K}$ is defined as

Definition 4. The optimal space of order $r$ is the space $\hat{P}_{r}^{o p t}(\hat{K})$ of minimal dimension on such that

$$
\hat{P}_{r}(\hat{K}) \supset \hat{P}_{r}^{o p t}(\hat{K}) \Leftrightarrow P_{r}^{F} \supset \mathfrak{D}_{r}(x, y, z)
$$

for any element $K$.

Theorem 2. The optimal spaces $\hat{P}_{r}^{o p t}$ are equal to:

\section{- Tetrahedron:}

$$
\hat{P}_{r}^{o p t}=\mathfrak{D}_{r}(\hat{x}, \hat{y}, \hat{z})
$$


- Hexahedron:

$$
\hat{P}_{r}^{o p t}=\mathfrak{Q}_{r}(\hat{x}, \hat{y}, \hat{z})=\mathbb{Q}_{r+2, r, r}(\hat{x}, \hat{y}, \hat{z}) \times \mathbb{Q}_{r, r+2, r}(\hat{x}, \hat{y}, \hat{z}) \times \mathbb{Q}_{r, r, r+2}(\hat{x}, \hat{y}, \hat{z})
$$

- Triangular prism:

$$
\hat{P}_{r}^{o p t}=\mathfrak{W}_{r}(\hat{x}, \hat{y}, \hat{z})=\left(\mathfrak{D}_{r+1}(\hat{x}, \hat{y}) \otimes \mathbb{P}_{r}(\hat{z})\right) \times \mathbb{W}_{r-1, r+2}(\hat{x}, \hat{y}, \hat{z})
$$

\section{- Pyramid:}

$$
\begin{aligned}
\hat{P}_{r}^{o p t}= & \mathfrak{B}_{r}(\hat{x}, \hat{y}, \hat{z})^{3} \\
= & \mathbb{B}_{r-1}(\hat{x}, \hat{y}, \hat{z})^{3} \\
& \left.\oplus\left[\begin{array}{c}
\frac{\hat{x}^{n+1} \hat{y}^{m}}{(1-\hat{z})^{m+1}} \\
0 \\
0
\end{array}\right] \oplus\left[\begin{array}{c}
0 \\
\frac{\hat{x}^{m} \hat{y}^{n+1}}{(1-\hat{z})^{m+1}} \\
0
\end{array}\right] 0 \leq m \leq n \leq r-1\right\} \\
& \oplus\left\{\frac{\hat{x}^{m} \hat{y}^{n+1}}{(1-\hat{z})^{m+1}}\left[\begin{array}{c}
\frac{\hat{x}}{(1-\hat{z})} \\
0 \\
-1
\end{array}\right] \oplus \frac{\hat{x}^{n+1} \hat{y}^{m}}{(1-\hat{z})^{m+1}}\left[\begin{array}{c}
0 \\
\frac{\hat{y}}{(1-\hat{z})} \\
-1
\end{array}\right] 0 \leq m \leq n \leq r-1\right\} \\
& \oplus\left\{\begin{array}{c}
\hat{x}^{i} \hat{y}^{j} \\
(1-\hat{z})^{i+j-r} \\
\frac{\hat{x}}{(1-\hat{z})} \\
\frac{\hat{y}}{(1-\hat{z})} \\
-1
\end{array}\right], 0 \leq i, j \leq r
\end{aligned}
$$

The optimal space for the pyramid is polynomial when expressed in the cube $[-1,1]^{2} \times[0,1]$ :

$$
\begin{aligned}
\hat{P}_{r}^{o p t} \circ T=\mathbb{C}_{r-1}^{3} & \oplus\left\{\left[\begin{array}{c}
\widetilde{x}^{n+1} \widetilde{y}^{m}(1-\widetilde{z})^{n} \\
0 \\
0
\end{array}\right], \quad\left[\begin{array}{c}
0 \\
\widetilde{x}^{m} \widetilde{y}^{n+1}(1-\widetilde{z})^{n} \\
0
\end{array}\right], \quad 0 \leq m \leq n \leq r-1\right\} \\
& \oplus\left\{\left[\begin{array}{c}
\widetilde{x}^{m+1} \widetilde{y}^{n+1}(1-\widetilde{z})^{n} \\
0 \\
-\widetilde{x}^{m} \widetilde{y}^{n+1}(1-\widetilde{z})^{n}
\end{array}\right], \quad\left[\begin{array}{c}
0 \\
\widetilde{x}^{n+1} \widetilde{y}^{m+1}(1-\widetilde{z})^{n} \\
-\widetilde{x}^{n+1} \widetilde{y}^{m}(1-\widetilde{z})^{n}
\end{array}\right], \quad 0 \leq m \leq n \leq r-1\right\} \\
& \oplus\left\{\widetilde{x}^{i} \widetilde{y}^{j}(1-\widetilde{z})^{r}\left[\begin{array}{c}
\widetilde{x} \\
\widetilde{y} \\
-1
\end{array}\right], \quad 0 \leq i, j \leq r\right\} .
\end{aligned}
$$

Proof. For any type of element, by construction, we obviously have

$$
\hat{E}_{r} \subset \hat{P}_{r}^{o p t} .
$$

The remaining polynomials to consider are the polynomials $p$ of $\widetilde{\mathbb{P}}_{r-1}\left[\begin{array}{l}x \\ y \\ z\end{array}\right]$ This part of the proof will be conducted for the pyramid and the hexahedron.

Let us begin with the pyramid. We consider a polynomial $p$ such that

$$
p=x^{i} y^{j} z^{r-1-i-j}\left[\begin{array}{l}
x \\
y \\
z
\end{array}\right]=\widetilde{x}^{i} \widetilde{y}^{j}(1-\widetilde{z})^{r-1}\left[\begin{array}{l}
x \\
y \\
z
\end{array}\right], \quad i, j \leq r-1
$$


by using the optimality of $\mathbb{B}_{r}$. The first component of $\hat{p}$ is equal to:

$$
\begin{aligned}
\hat{p}_{x}= & \widetilde{x}^{i} \widetilde{y}^{j}(1-\widetilde{z})^{r-1} \operatorname{comat}(D F)_{x} \cdot F= \\
& \widetilde{x}^{i} \widetilde{y}^{j}(1-\widetilde{z})^{r-1}\left(A_{2} \times A_{3}+C \times A_{3} \widetilde{x}+A_{2} \times C \widetilde{x} \widetilde{y}\right) \cdot\left(A_{0}+A_{3}+A_{1} \widetilde{x}(1-\widetilde{z})+A_{2} \widetilde{y}(1-\widetilde{z})\right. \\
& \left.\quad-A_{3}(1-\widetilde{z})+C \widetilde{x} \widetilde{y}(1-\widetilde{z})\right) .
\end{aligned}
$$

If we note $\bar{A}_{0}=A_{0}+A_{3}$, we obtain:

$$
\begin{aligned}
\hat{p}_{x}= & \widetilde{x}^{i} \widetilde{y}^{j}(1-\widetilde{z})^{r-1}\left[\left(A_{2}, A_{3}, \bar{A}_{0}\right)+\left(A_{2}, A_{3}, A_{1}\right) \widetilde{x}(1-\widetilde{z})+\left(C, A_{3}, \bar{A}_{0}\right) \widetilde{x}\right. \\
& \left.+\left(C, A_{3}, A_{1}\right) \widetilde{x}^{2}(1-\widetilde{z})+\left(A_{2}, C, \bar{A}_{0}\right) \widetilde{x} \widetilde{y}+\left(A_{2}, C, A_{1}\right) \widetilde{x}^{2} \widetilde{y}(1-\widetilde{z})-\left(A_{2}, C, A_{3}\right) \widetilde{x} \widetilde{y}(1-\widetilde{z})\right] .
\end{aligned}
$$

Similar computations give the following values for the second and third component of $\hat{p}$

$$
\begin{aligned}
\hat{p}_{y}= & \widetilde{x}^{i} \widetilde{y}^{j}(1-\widetilde{z})^{r-1}\left[\left(A_{3}, A_{1}, \bar{A}_{0}\right)+\left(A_{3}, A_{1}, A_{2}\right) \widetilde{y}(1-\widetilde{z})+\left(A_{3}, C, \bar{A}_{0}\right) \widetilde{y}\right. \\
& \left.+\left(A_{3}, C, A_{2}\right) \widetilde{y}^{2}(1-\widetilde{z})+\left(C, A_{1}, \bar{A}_{0}\right) \widetilde{x} \widetilde{y}+\left(C, A_{1}, A_{2}\right) \widetilde{x} \widetilde{y}^{2}(1-\widetilde{z})-\left(C, A_{1}, A_{3}\right) \widetilde{x} \widetilde{y}(1-\widetilde{z})\right] \\
\hat{p}_{z}= & \widetilde{x}^{i} \widetilde{y}^{j}(1-\widetilde{z})^{r-1}\left[\left(A_{1}, A_{2}, \bar{A}_{0}\right)-\left(A_{1}, A_{2}, A_{3}\right)(1-\widetilde{z})+\left(A_{1}, C, \bar{A}_{0}\right) \widetilde{x}-\left(A_{1}, C, A_{3}\right) \widetilde{x}(1-\widetilde{z})\right. \\
& \left.+\left(C, A_{2}, \bar{A}_{0}\right) \widetilde{y}+\left(C, A_{2}, A_{1}\right) \widetilde{x} \widetilde{y}(1-\widetilde{z})-\left(C, A_{2}, A_{3}\right) \widetilde{y}(1-\widetilde{z})\right] .
\end{aligned}
$$

The terms involving $\bar{A}_{0}$ can be removed since they have already been treated when considering $p$ in $\mathbb{P}_{r-1}^{3}$. It remains the following monomials:

$$
\begin{aligned}
\widetilde{x}^{i} \widetilde{y}^{j}(1-\widetilde{z})^{r-1}( & \left(A_{1}, A_{2}, A_{3}\right)\left[\begin{array}{c}
\widetilde{x}(1-\widetilde{z}) \\
\widetilde{y}(1-\widetilde{z}) \\
-(1-\widetilde{z})
\end{array}\right]+\left(C, A_{3}, A_{1}\right)\left[\begin{array}{c}
\widetilde{x}^{2}(1-\widetilde{z}) \\
\widetilde{x} \widetilde{y}(1-\widetilde{z}) \\
-\widetilde{x}(1-\widetilde{z})
\end{array}\right] \\
& \left.+\left(C, A_{1}, A_{2}\right)\left[\begin{array}{c}
\widetilde{x}^{2} \widetilde{y}(1-\widetilde{z}) \\
\widetilde{x} \widetilde{y}^{2}(1-\widetilde{z}) \\
-\widetilde{x} \widetilde{y}(1-\widetilde{z})
\end{array}\right]+\left(C, A_{2}, A_{3}\right)\left[\begin{array}{c}
\widetilde{x} \widetilde{y}(1-\widetilde{z}) \\
\widetilde{y}^{2}(1-\widetilde{z}) \\
-\widetilde{y}(1-\widetilde{z})
\end{array}\right]\right), \quad i, j \leq r-1 .
\end{aligned}
$$

We notice that the coefficients $\left(A_{1}, A_{2}, A_{3}\right),\left(C, A_{3}, A_{1}\right),\left(A_{2}, C, A_{1}\right),\left(C, A_{2}, A_{3}\right)$ viewed as functions of the vertices of the pyramid $S_{0}, S_{1}, S_{2}, S_{3}, S_{4}$ are linearly independent, then all the monomials are needed. These monomials can be regrouped in a single family:

$$
\left\{\widetilde{x}^{i} \widetilde{y}^{j}(1-\widetilde{z})^{r}\left[\begin{array}{c}
\widetilde{x} \\
\widetilde{y} \\
-1
\end{array}\right], \quad i, j \leq r\right\} .
$$

We recognize here the last family of the optimal space of the pyramid, the other families coming directly from $\hat{E}_{r}$.

Let us consider a general hexahedron, and the polynomial $p$ :

$$
p=\left[\begin{array}{l}
x \\
y \\
z
\end{array}\right]
$$

Because of Piola transform, the first component will be equal to

$$
\begin{aligned}
\hat{p}_{x}= & \left(A_{2} \times A_{3}+\hat{x}\left(C_{1} \times A_{3}+A_{2} \times C_{2}\right)+\hat{y} A_{2} \times C_{3}+\hat{z} C_{3} \times A_{3}+\hat{x} \hat{y}\left(A_{2} \times D+C_{1} \times C_{3}\right)\right. \\
& \left.+\hat{x} \hat{z}\left(D \times A_{3}+C_{3} \times C_{2}\right)+\hat{x}^{2} C_{1} \times C_{2}+\hat{x}^{2} \hat{y} C_{1} \times D+\hat{x}^{2} \hat{z} D \times C_{2}\right) \cdot\left(A_{0}+\hat{x} A_{1}+\hat{y} A_{2}+\hat{z} A_{3}\right. \\
& \left.+\hat{x} \hat{y} C_{1}+\hat{x} \hat{z} C_{2}+\hat{y} \hat{z} C_{3}+\hat{x} \hat{y} \hat{z} D\right) .
\end{aligned}
$$


By expanding this expression, and computing the two other components of $\hat{p}$, we find the following result

$$
\begin{aligned}
& \hat{p}=\left(A_{1}, A_{2}, C_{1}\right)\left[\begin{array}{c}
0 \\
0 \\
-\hat{x} \hat{y}
\end{array}\right]+\left(A_{1}, A_{2}, C_{2}\right)\left[\begin{array}{c}
\hat{x}^{2} \\
\hat{x} \hat{y} \\
0
\end{array}\right]+\left(A_{1}, A_{2}, C_{3}\right)\left[\begin{array}{c}
\hat{x} \hat{y} \\
\hat{y}^{2} \\
0
\end{array}\right]+\left(A_{1}, A_{2}, D\right)\left[\begin{array}{c}
\hat{x}^{2} \hat{y} \\
\hat{x} \hat{y}^{2} \\
-\hat{x} \hat{y} \hat{z}
\end{array}\right] \\
& +\left(A_{1}, A_{3}, C_{1}\right)\left[\begin{array}{c}
-\hat{x}^{2} \\
0 \\
-\hat{x} \hat{z}
\end{array}\right]+\left(A_{1}, A_{3}, C_{2}\right)\left[\begin{array}{c}
0 \\
\hat{x} \hat{z} \\
0
\end{array}\right]+\left(A_{1}, A_{3}, C_{3}\right)\left[\begin{array}{c}
-\hat{x} \hat{z} \\
0 \\
-\hat{z}^{2}
\end{array}\right]+\left(A_{1}, A_{3}, D\right)\left[\begin{array}{c}
\hat{x}^{2} \hat{z} \\
-\hat{x} \hat{y} \hat{z} \\
\hat{x} \hat{z}^{2}
\end{array}\right] \\
& +\left(A_{2}, A_{3}, C_{1}\right)\left[\begin{array}{c}
0 \\
\hat{y}^{2} \\
\hat{y} \hat{z}
\end{array}\right]+\left(A_{2}, A_{3}, C_{2}\right)\left[\begin{array}{c}
0 \\
\hat{y} \hat{z} \\
\hat{z}^{2}
\end{array}\right]+\left(A_{2}, A_{3}, C_{3}\right)\left[\begin{array}{c}
\hat{y} \hat{z} \\
0 \\
0
\end{array}\right]+\left(A_{2}, A_{3}, D\right)\left[\begin{array}{c}
\hat{x} \hat{y} \hat{z} \\
-\hat{y}^{2} \hat{z} \\
-\hat{y} \hat{z}^{2}
\end{array}\right] \\
& +\left(A_{1}, C_{1}, C_{2}\right)\left[\begin{array}{c}
\hat{x}^{3} \\
0 \\
0
\end{array}\right]+\left(A_{1}, C_{1}, C_{3}\right)\left[\begin{array}{c}
\hat{x}^{2} \hat{y} \\
0 \\
\hat{x} \hat{y} \hat{z}
\end{array}\right]+\left(A_{1}, C_{2}, C_{3}\right)\left[\begin{array}{c}
-\hat{x}^{2} \hat{z} \\
-\hat{x} \hat{y} \hat{z} \\
0
\end{array}\right]+\left(A_{2}, C_{1}, C_{2}\right)\left[\begin{array}{c}
0 \\
-\hat{x} \hat{y}^{2} \\
-\hat{x} \hat{y} \hat{z}
\end{array}\right] \\
& +\left(A_{2}, C_{1}, C_{3}\right)\left[\begin{array}{c}
0 \\
-\hat{y}^{3} \\
0
\end{array}\right]+\left(A_{2}, C_{2}, C_{3}\right)\left[\begin{array}{c}
-\hat{x} \hat{y} \hat{z} \\
-\hat{y}^{2} \hat{z} \\
0
\end{array}\right]+\left(A_{3}, C_{1}, C_{2}\right)\left[\begin{array}{c}
0 \\
-\hat{x} \hat{y} \hat{z} \\
-\hat{x} \hat{z}^{2}
\end{array}\right]+\left(A_{3}, C_{1}, C_{3}\right)\left[\begin{array}{c}
\hat{x} \hat{y} \hat{z} \\
0 \\
\hat{y} \hat{z}^{2}
\end{array}\right] \\
& +\left(A_{3}, C_{2}, C_{3}\right)\left[\begin{array}{c}
0 \\
0 \\
\hat{z}^{3}
\end{array}\right]+\left(A_{1}, C_{1}, D\right)\left[\begin{array}{c}
\hat{x}^{3} \hat{y} \\
0 \\
0
\end{array}\right]+\left(A_{1}, C_{2}, D\right)\left[\begin{array}{c}
-\hat{x}^{3} \hat{z} \\
0 \\
0
\end{array}\right]+\left(A_{1}, C_{3}, D\right)\left[\begin{array}{c}
0 \\
\hat{x} \hat{y}^{2} \hat{z} \\
-\hat{x} \hat{y} \hat{z}^{2}
\end{array}\right] \\
& +\left(A_{2}, C_{1}, D\right)\left[\begin{array}{c}
0 \\
-\hat{x} \hat{y}^{3} \\
0
\end{array}\right]+\left(A_{2}, C_{2}, D\right)\left[\begin{array}{c}
-\hat{x}^{2} \hat{y} \hat{z} \\
0 \\
\hat{x} \hat{y} \hat{z}^{2}
\end{array}\right]+\left(A_{2}, C_{3}, D\right)\left[\begin{array}{c}
0 \\
\hat{y}^{3} \hat{z} \\
0
\end{array}\right]+\left(A_{3}, C_{1}, D\right)\left[\begin{array}{c}
\hat{x}^{2} \hat{y} \hat{z} \\
-\hat{x} \hat{y}^{2} \hat{z} \\
0
\end{array}\right] \\
& +\left(A_{3}, C_{2}, D\right)\left[\begin{array}{c}
0 \\
0 \\
\hat{x} \hat{z}^{3}
\end{array}\right]+\left(A_{3}, C_{3}, D\right)\left[\begin{array}{c}
0 \\
0 \\
-\hat{y} \hat{z}^{3}
\end{array}\right]+\left(C_{1}, C_{2}, C_{3}\right)\left[\begin{array}{c}
\hat{x} \hat{y} \hat{z} \\
\hat{x} \hat{y}^{2} \hat{z} \\
\hat{x} \hat{y} \hat{z}^{2}
\end{array}\right]+\left(C_{1}, C_{2}, D\right)\left[\begin{array}{c}
-\hat{x}^{3} \hat{y} \hat{z} \\
0 \\
0
\end{array}\right] \\
& +\left(C_{1}, C_{3}, D\right)\left[\begin{array}{c}
0 \\
\hat{x} \hat{y}^{3} \hat{z} \\
0
\end{array}\right]+\left(C_{2}, C_{3}, D\right)\left[\begin{array}{c}
0 \\
0 \\
-\hat{x} \hat{y} \hat{z}^{3}
\end{array}\right]+\text { other terms in } \mathbb{P}_{1}^{3} \text {. }
\end{aligned}
$$

Since the coefficients $\left(A_{1}, A_{2}, A_{3}\right),\left(A_{1}, A_{2}, C_{1}\right), \ldots$ are linearly independent, all the monomials written above are needed to generate $\mathfrak{D}_{r}$. When we combine these monomials with monomials of $\hat{E}_{1}$, we can observe that we obtain exactly

$$
\mathbb{Q}_{3,1,1} \times \mathbb{Q}_{1,3,1} \times \mathbb{Q}_{1,1,3}
$$

For any $r$, since we have to multiply by the polynomials $\hat{x}^{i} \hat{y}^{j} \hat{z}^{k}$ with $0 \leq i, j, k \leq r-1$, we obtain that:

$$
\hat{P}_{r}^{o p t}=\mathbb{Q}_{r+2, r, r} \times \mathbb{Q}_{r, r+2, r} \times \mathbb{Q}_{r, r, r+2} .
$$

Remark 1. The dimension of the optimal spaces is equal to

Tetrahedron: $\operatorname{dim} \hat{P}_{r}^{o p t}=\frac{r(r+1)(r+3)}{2}$

Hexahedron: $\operatorname{dim} \hat{P}_{r}^{o p t}=3(r+3)(r+1)^{2}$

Triangular prisms: $\operatorname{dim} \hat{P}_{r}^{o p t}=\frac{(r+1)(r+3)(3 r+2)}{2}$

Pyramids: $\operatorname{dim} \hat{P}_{r}^{o p t}=\frac{(r+1)\left(2 r^{2}+7 r+2\right)}{2}$

\subsection{Super-Optimal Finite Element Spaces}

The needed and sufficient conditions in order to obtain optimal error estimates for $H(\mathrm{div})$ are the following ones (see (10)):

$$
\left\{\begin{array}{l}
P_{r}^{F} \supset \mathbb{P}_{r-1}(x, y, z)^{3} \\
\operatorname{div} P_{r}^{F} \supset \mathbb{P}_{r-1}(x, y, z) .
\end{array}\right.
$$


The optimal spaces previously constructed verify these properties since div $\mathfrak{D}_{r}=\mathbb{P}_{r-1}$, but spaces of lower dimension satisfying to these conditions can be constructed for each type of element.

We first define the following space:

Definition 5. The space $\hat{F}_{r}$ of order $r$ is the space of minimal dimension on $\hat{K}$ such that

$$
\operatorname{div} \hat{P}_{r}(\hat{K}) \supset \hat{F}_{r} \Leftrightarrow \operatorname{div} P_{r}^{F} \supset \mathbb{P}_{r-1}(x, y, z)
$$

for any element $K$.

Theorem 3. The spaces $\hat{F}_{r}$ are equal to:

- Tetrahedron:

$$
\hat{F}_{r}=\mathbb{P}_{r-1}(\hat{x}, \hat{y}, \hat{z})
$$

- Hexahedron:

$$
\hat{F}_{r}=\mathbb{Q}_{r, r, r}(\hat{x}, \hat{y}, \hat{z}) \oplus\left\{\hat{x}^{r+1} \hat{y}^{m} \hat{z}^{n}, \hat{x}^{m} \hat{y}^{r+1} \hat{z}^{n}, \hat{x}^{m} \hat{y}^{n} \hat{z}^{r+1}, \quad 0 \leq m, n \leq r\right\}
$$

\section{- Triangular prism:}

$$
\hat{F}_{r}=\mathbb{W}_{r, r}(\hat{x}, \hat{y}, \hat{z}) \oplus \mathbb{P}_{r-1}(\hat{x}, \hat{y}) \hat{z}^{r+1}
$$

\section{- Pyramid:}

$$
\hat{F}_{r}=\left\{\begin{array}{ll}
\hat{x}^{i} \hat{y}^{j} & 0 \leq i, j \leq k+1 \\
(1-\hat{z})^{i+j-k} & 0 \leq k \leq r-1
\end{array}\right\}
$$

Proof. We complete the proof for the pyramid and the hexahedron. Let us consider a function $p \in P_{r}^{F}$, such that

$$
\operatorname{div} p=x^{i} y^{j} z^{k}, i+j+k \leq r-1
$$

For the pyramid, because of the optimality of $\mathbb{C}_{r-1}$, it is equivalent to consider a function such that

$$
\operatorname{div} p=\widetilde{x}^{i} \widetilde{y}^{j}(1-\widetilde{z})^{k}, i, j \leq k \leq r-1
$$

We use again coordinates $(\widetilde{x}, \widetilde{y}, \widetilde{z})$ of the symmetric cube $[-1,1]^{2} \times[0,1]$. We have the relationship

$$
\operatorname{div} \hat{p}=|D F| p \text {. }
$$

The jacobian $|D F|$ is equal to:

$|D F|=\left(A_{1}+C \widetilde{y}\right) \times\left(A_{2}+C \widetilde{x}\right) \cdot\left(A_{3}+C \widetilde{x} \widetilde{y}\right)=\left(A_{1}, A_{2}, A_{3}\right)+\left(A_{1}, C, A_{3}\right) \widetilde{x}+\left(C, A_{2}, A_{3}\right) \widetilde{y}+\left(A_{1}, A_{2}, C\right) \widetilde{x} \widetilde{y}$

Since the constants $\left(A_{1}, A_{2}, A_{3}\right),\left(A_{1}, C, A_{3}\right),\left(C, A_{2}, A_{3}\right),\left(A_{1}, A_{2}, C\right)$ viewed as functions of $A_{1}, A_{2}, A_{3}, C$ are linearly independent, the following monomials are necessarily included in $F_{r}$ :

$$
\widetilde{x}^{i} \widetilde{y}^{j}(1-\widetilde{z})^{k}, \widetilde{x}^{i+1} \widetilde{y}^{j}(1-\widetilde{z})^{k}, \widetilde{x}^{i} \widetilde{y}^{j+1}(1-\widetilde{z})^{k}, \widetilde{x}^{i+1} \widetilde{y}^{j+1}(1-\widetilde{z})^{k} \text {. }
$$

For instance, the last monomial gives:

$$
\widetilde{x}^{i+1} \widetilde{y}^{j+1}(1-\widetilde{z})^{k}=\frac{\hat{x}^{i+1} \hat{y}^{j+1}}{(1-\hat{z})^{i+1+j+1-k}} .
$$

By naming $i^{\prime}=i+1, j^{\prime}=j+1$, we obtain:

$$
\widetilde{x}^{i+1} \widetilde{y}^{j+1}(1-\widetilde{z})^{k}=\frac{\hat{x}^{i^{\prime}} \hat{y}^{j^{\prime}}}{(1-\hat{z})^{i^{\prime}+j^{\prime}-k}}, i^{\prime} \leq j^{\prime} \leq k+1
$$


which corresponds to a function of $\hat{F}_{r}$. It is immediate that these monomials will generate all the functions of $\hat{F}_{r}$.

For the hexahedron, we consider a function $p$ such that

$$
\operatorname{div} p=\hat{x}^{i} \hat{y}^{j} \hat{z}^{k}, i, j, k \leq r-1 .
$$

Similarly to the pyramid, the jacobian $|D F|$ of the hexahedron can be expressed as

$$
\begin{aligned}
|D F|= & b_{0}+b_{1} \hat{x}+b_{2} \hat{y}+b_{3} \hat{z}+b_{4} \hat{x} \hat{y}+b_{5} \hat{x} \hat{z}+b_{6} \hat{y} \hat{z}+b_{7} \hat{x} \hat{y} \hat{z}+b_{8} \hat{x}^{2}+b_{9} \hat{x}^{2} \hat{y}+b_{10} \hat{x}^{2} \hat{z}+b_{11} \hat{x}^{2} \hat{y} \hat{z}+ \\
& b_{12} \hat{y}^{2}+b_{13} \hat{x} \hat{y}^{2}+b_{14} \hat{y}^{2} \hat{z}+b_{15} \hat{x} \hat{y}^{2} \hat{z}+b_{16} \hat{z}^{2}+b_{17} \hat{x} \hat{z}^{2}+b_{18} \hat{y} \hat{z}^{2}+b_{19} \hat{x} \hat{y} \hat{z}^{2}
\end{aligned}
$$

with linearly independent functions $b_{0}, b_{1}, \cdots, b_{19}$. Therefore all the functions of $\mathbb{Q}_{r+1, r, r}+\mathbb{Q}_{r, r+1, r}+\mathbb{Q}_{r, r, r+1}$ are necessary and sufficient to generate any $p$ such that $\operatorname{div} p \in \mathbb{P}_{r-1}(x, y, z)$. We obtain the claimed space $\hat{F}_{r}$ for the hexahedron.

Proposition 1. The divergence of the spaces $\hat{E}_{r}$ is equal to

$$
\begin{aligned}
& \text { Tetrahedron: } \quad \operatorname{div} \hat{E}_{1}=0 \\
& \operatorname{div} \hat{E}_{r}=\mathbb{P}_{r-2}(\hat{x}, \hat{y}, \hat{z}), r>1 \\
& \operatorname{div} \hat{E}_{r}=\mathbb{W}_{r-2, r}(\hat{x}, \hat{y}, \hat{z}) \oplus \widetilde{\mathbb{P}}_{r-1}(\hat{x}, \hat{y}) \mathbb{P}_{r-1}(\hat{z}), r>1
\end{aligned}
$$
Hexahedron: $\quad \operatorname{div} \hat{E}_{1}=\operatorname{Span}\{1\}$ $\operatorname{div} \hat{E}_{r}=\hat{F}_{r-1}, r>1$
Pyramid: $\quad \operatorname{div} \hat{E}_{r}=\hat{F}_{r-1} \oplus\left\{\frac{1}{1-\hat{z}}\right\}$ with $F_{0}=\varnothing$

Proof. The proof is immediate by computing the divergence of all monomials of $\hat{E}_{r}$. It should be noticed that $\frac{1}{1-\hat{z}}$ for the pyramid is obtained as the divergence of the function

$$
\frac{\hat{x}^{n+1} \hat{y}^{m}}{(1-\hat{z})^{m+1}}\left[\begin{array}{l}
1 \\
0 \\
0
\end{array}\right]
$$

for the special case $m=n=0$. Other values of $m, n$ give a divergence in $\hat{F}_{r-1}$. The divergence of the functions of the last family appearing in the expression of $\hat{E}_{r}$ is null.

Now we have all the ingredients needed to find the super-optimal finite element spaces.

Definition 6. A super-optimal space of order $r$ is a space $\hat{P}_{r}^{s-o p t}(\hat{K})$ of minimal dimension on such that

$$
\hat{P}_{r}(\hat{K}) \supset \hat{P}_{r}^{s-o p t} \Rightarrow\left\{\begin{array}{l}
P_{r}^{F} \supset \mathbb{P}_{r-1}(x, y, z)^{3} \\
\operatorname{div} P_{r}^{F} \supset \mathbb{P}_{r-1}(x, y, z)
\end{array}\right.
$$

for any element $K$.

Theorem 4. A set of super-optimal spaces $\hat{P}_{r}^{s-o p t}$ is equal to:

\section{- Tetrahedron:}

$$
\hat{P}_{r}^{s-o p t}=\hat{P}_{r}^{o p t}
$$


- Hexahedron:

$$
\begin{aligned}
\hat{P}_{r}^{s-o p t}= & \left\{\left[\begin{array}{c}
\hat{x}^{i} \hat{y}^{j} \hat{z}^{k} \\
0 \\
0
\end{array}\right],\left[\begin{array}{c}
0 \\
\hat{x}^{j} \hat{y}^{i} \hat{z}^{k} \\
0
\end{array}\right],\left[\begin{array}{c}
0 \\
0 \\
\hat{x}^{j} \hat{y}^{k} \hat{z}^{i}
\end{array}\right], \quad 0 \leq i \leq r+1,0 \leq j, k \leq r-1\right\} \\
& \oplus\left\{\left[\begin{array}{c}
\hat{x}^{i} \hat{y}^{j} \hat{z}^{k} \\
0 \\
0
\end{array}\right],\left[\begin{array}{c}
0 \\
\hat{x}^{j} \hat{y}^{i} \hat{z}^{k} \\
0
\end{array}\right],\left[\begin{array}{c}
0 \\
0 \\
\hat{x}^{j} \hat{y}^{k} \hat{z}^{i}
\end{array}\right], \quad i=r+2,0 \leq j, k \leq r\right\} \\
& \oplus\left\{\left[\begin{array}{c}
\hat{x}^{i} \hat{y}^{r} \hat{z}^{k} \\
0 \\
0
\end{array}\right],\left[\begin{array}{c}
0 \\
\hat{x}^{r} \hat{y}^{i} \hat{z}^{k} \\
0
\end{array}\right],\left[\begin{array}{c}
0 \\
0 \\
\hat{x}^{r} \hat{y}^{k} \hat{z}^{i}
\end{array}\right], \quad 0 \leq i \leq r+1, \quad 0 \leq k \leq r-1\right\} \\
& \oplus\left\{\left[\begin{array}{c}
\hat{x}^{i} \hat{y}^{k} \hat{z}^{r} \\
0 \\
0
\end{array}\right],\left[\begin{array}{c}
0 \\
0 \\
\hat{x}^{k} \hat{y}^{i} \hat{z}^{r} \\
0
\end{array}\right],\left[\begin{array}{c}
0 \\
\hat{x}^{k} \hat{y}^{r} \hat{z}^{i}
\end{array}\right], \quad 0 \leq i \leq r+1, \quad 0 \leq k \leq r-1\right\} \\
& \oplus\left\{\left[\begin{array}{c}
\hat{x}^{r+1} \hat{y}^{r} \hat{z}^{r} \\
\hat{x}^{r} \hat{y}^{r+1} \hat{z}^{r} \\
\hat{x}^{r} \hat{y}^{r} \hat{z}^{r+1}
\end{array}\right]\right\}
\end{aligned}
$$

- Triangular prism:

$$
\hat{P}_{r}^{s-o p t}=\hat{P}_{r}^{o p t} \backslash\left\{\left(\widetilde{\mathbb{P}}_{r}(\hat{x}, \hat{y}) \hat{z}^{r}\right)^{2} \times\{0\}\right\} \oplus \widetilde{\mathbb{P}}_{r-1}(\hat{x}, \hat{y}) \hat{z}^{r}\left[\begin{array}{l}
\hat{x} \\
\hat{y} \\
0
\end{array}\right]
$$

- Pyramid:

$$
\hat{P}_{r}^{s-o p t}=\hat{P}_{r}^{o p t}
$$

Proof. We make the proof only for the hexahedron. For $r=1$, it suffices to examine the 21 monomials of $\hat{E}_{1}$ and the 19 monomials of $\hat{F}_{1} \backslash\{1\}$ to check that $\hat{P}_{1}^{s-o p t}$ satisfies the appropriate conditions, and is minimal since its dimension is equal to 40 . For $r>1$, since div $\hat{E}_{r}=\hat{F}_{r-1}$, the condition

$$
\operatorname{div} \hat{P}_{r}^{s-o p t} \supset \hat{F}_{r}
$$

is equivalent to

$$
\operatorname{div} \hat{P}_{r}^{s-o p t} \supset \hat{F}_{r} \backslash \hat{F}_{r-1} .
$$

This last condition is much more interesting, since it will not overlap with the condition

$$
\hat{P}_{r} \supset \hat{E}_{r} .
$$

Therefore, a minimal space will have the following form:

$$
\hat{P}_{r}^{s-o p t}=\hat{E}_{r} \oplus \hat{G}_{r}
$$

with

$$
\operatorname{div} \hat{G}_{r}=\hat{F}_{r} \backslash \hat{F}_{r-1} .
$$


We see here that $\hat{G}_{r}$ cannot be uniquely determined by this relation, an infinite number of spaces will satisfy this relation. That's why the optimal space $\hat{P}_{r}^{s-o p t}$ is not unique. In order to choose a space, we have applied the following rule:

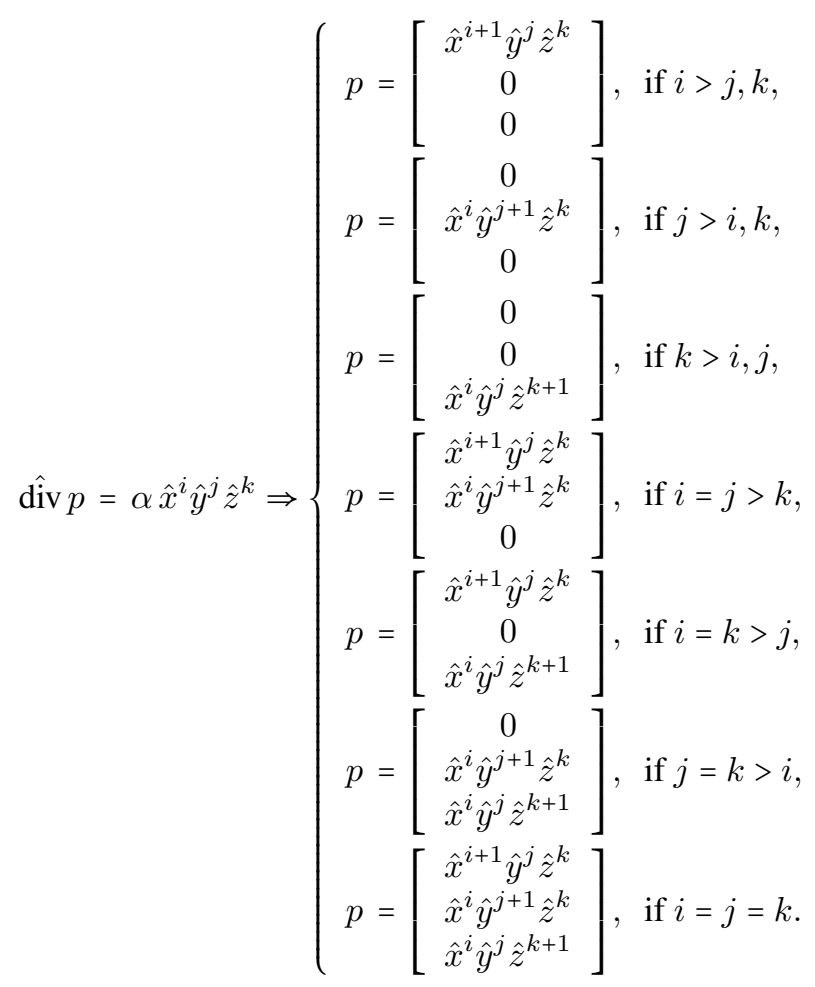

By selecting this rule, the obtained optimal space keeps all the symmetries in $x, y, z$. We have:

$$
\begin{aligned}
& \hat{F}_{r} \backslash \hat{F}_{r-1}=\left\{\hat{x}^{r} \hat{y}^{r} \hat{z}^{i}, \hat{x}^{r} \hat{y}^{i} \hat{z}^{r}, \hat{x}^{i} \hat{y}^{r} \hat{z}^{r}, \quad i \leq r-1\right\} \\
& \oplus\left\{\hat{x}^{r} \hat{y}^{r} \hat{z}^{r}\right\} \oplus\left\{\hat{x}^{r+1} \hat{y}^{i} \hat{z}^{j}, \hat{x}^{i} \hat{y}^{r+1} \hat{z}^{j}, \hat{x}^{i} \hat{y}^{j} \hat{z}^{r+1}, \quad i, j \leq r\right\} .
\end{aligned}
$$

With our rule, we obtain:

$$
\begin{aligned}
\hat{G}_{r}= & \left\{\left[\begin{array}{c}
\hat{x}^{r+1} \hat{y}^{r} \hat{z}^{i} \\
\hat{x}^{r} \hat{y}^{r+1} \hat{z}^{i} \\
0
\end{array}\right],\left[\begin{array}{c}
\hat{x}^{r+1} \hat{y}^{i} \hat{z}^{r} \\
0 \\
\hat{x}^{r} \hat{y}^{i} \hat{z}^{r+1}
\end{array}\right],\left[\begin{array}{c}
0 \\
\hat{x}^{i} \hat{y}^{r+1} \hat{z}^{r} \\
\hat{x}^{i} \hat{y}^{r} \hat{z}^{r+1}
\end{array}\right], \quad i \leq r-1\right\} \\
& \oplus\left\{\left[\begin{array}{c}
\hat{x}^{r+1} \hat{y}^{r} \hat{z}^{r} \\
\hat{x}^{r} \hat{y}^{r+1} \hat{z}^{r} \\
\hat{x}^{r} \hat{y}^{r} \hat{z}^{r+1}
\end{array}\right]\right\} \oplus\left\{\left[\begin{array}{c}
\hat{x}^{r+2} \hat{y}^{i} \hat{z}^{j} \\
0 \\
0
\end{array}\right],\left[\begin{array}{c}
0 \\
\hat{x}^{i} \hat{y}^{r+2} \hat{z}^{j} \\
0
\end{array}\right],\left[\begin{array}{c}
0 \\
0 \\
\hat{x}^{i} \hat{y}^{j} \hat{z}^{r+2}
\end{array}\right], \quad i, j \leq r\right\} .
\end{aligned}
$$

Since we have

$$
\left[\begin{array}{c}
\hat{x}^{r+1} \hat{y}^{r} \hat{z}^{i} \\
\hat{x}^{r} \hat{y}^{r+1} \hat{z}^{i} \\
0
\end{array}\right] \in \hat{G}_{r} \text { and }\left[\begin{array}{c}
\hat{x}^{r+1} \hat{y}^{r} \hat{z}^{i} \\
-\hat{x}^{r} \hat{y}^{+1} \hat{z}^{i} \\
0
\end{array}\right] \in \hat{E}_{r}
$$

we obtain by summation $\left(\hat{x}^{r+1} \hat{y}^{r} \hat{z}^{i}, 0,0\right) \in P_{r}^{o p t}$, and symmetric monomials in $\hat{y}, \hat{z}$. By merging all the other monomials of $\hat{G}_{r}$ and $\hat{E}_{r}$, we obtain the claimed expression of $\hat{P}_{r}^{s-o p t}$.

Remark 2. Since the divergence of two different spaces can be the same, the super-optimal spaces are not unique, an infinity of super optimal spaces with a minimal dimension do exist. We have given here a set of super-optimal spaces. In the numerical experiments the basis functions chosen for the hexahedron (detailed in Proposition 2) will not span the proposed space, but an equivalent one of the same dimension. 
Remark 3. The dimension of the super-optimal spaces is equal to

Tetrahedron: $\operatorname{dim} \hat{P}_{r}^{s-o p t}=\operatorname{dim} \hat{P}_{r}^{o p t} \quad$ Hexahedron: $\operatorname{dim} \hat{P}_{r}^{s-o p t}=\operatorname{dim} \hat{P}_{r}^{o p t}-(5+3 r)$ Pyramids: $\operatorname{dim} \hat{P}_{r}^{s-o p t}=\operatorname{dim} \hat{P}_{r}^{o p t} \quad$ Triangular prisms: $\operatorname{dim} \hat{P}_{r}^{s-o p t}=\operatorname{dim} \hat{P}_{r}^{o p t}-(2+r)$

The dimension of optimal finite element spaces is very close to the dimension of super-optimal finite element spaces. Therefore, since the optimal spaces do not include any linked function and has an attractive tensorized structure, these spaces seem more suitable for the numerical computations.

\subsection{Restriction of Normal Traces on the Faces}

To ensure the compatibility between elements, it is essential that the restriction of normal components of the functions span the same space on triangular and quadrilateral faces. A simple computation provides the following theorem:

Theorem 5. The normal traces are equal to:

For the optimal finite element spaces $\hat{P}_{r}^{o p t}$

$$
\begin{aligned}
& \text { For the super-optimal finite element spaces } \hat{P}_{r}^{s-o p t} \\
& \qquad \begin{array}{l}
\mathbb{Q}_{r, r}(x, y) \backslash\left\{x^{r} y^{r}\right\} \text { for quadrilateral faces } \\
\mathbb{P}_{r-1}(x, y) \text { for triangular faces }
\end{array}
\end{aligned}
$$$$
\mathbb{Q}_{r, r}(x, y) \text { for quadrilateral faces }
$$$$
\mathbb{P}_{r-1}(x, y) \text { for triangular faces }
$$

Proof. The proof is completed by computing for each monomial $p$ of $\hat{P}_{r}^{o p t}$ the quantity $p \cdot n$ on each face of the element, where $n$ is the normale.

\section{De Rham Diagram}

The stability for $H(c u r l)$ and $H(d i v)$ conforming elements comes from the exact sequence of De Rham diagram (see Monk (15)) which is directly linked to Helmholtz decomposition

$$
\begin{aligned}
& H^{1} \stackrel{\text { grad }}{\longrightarrow} H(\text { curl }) \stackrel{\text { curl }}{\longrightarrow} H(\text { div }) \stackrel{\text { div }}{\longrightarrow} L^{2} \\
& \cup \cup \cup U \\
& W_{r}^{1} \stackrel{\text { grad }}{\longrightarrow} W_{r}^{\text {curl }} \stackrel{\text { curl }}{\longrightarrow} W_{r}^{\text {div }} \stackrel{\text { div }}{\longrightarrow} W_{r}^{2}
\end{aligned}
$$

where $W_{r}^{H 1}, W_{r}^{\text {curl }}, W_{r}^{\text {div }}$ and $W_{r}^{2}$ are the spaces of order $r$ discretizing respectively $H^{1}(\Omega), H($ curl,$\Omega), H($ div,$\Omega)$ and $L^{2}(\Omega)$ (see Demkowicz (16)). The approximation spaces must then verify

$$
\begin{aligned}
& \operatorname{Im} \operatorname{grad} W_{r+1}^{1}=\operatorname{Ker} W_{r}^{\text {curl }}=\left\{u \in W_{r}^{\text {curl }} \mid \text { curl } u=0\right\} \\
& \operatorname{Im} \text { curl } W_{r}^{\text {curl }}=\operatorname{Ker} W_{r}^{\text {div }}=\left\{u \in W_{r}^{\text {div }} \mid \text { div } u=0\right\} \\
& \operatorname{Im} \operatorname{div} W_{r}^{\text {div }}=\operatorname{Ker} W_{r}^{2}=W_{r}^{2}
\end{aligned}
$$

for the four types of elements. (17) proposed a formalism to construct tetrahedra, hexahedra and triangular prisms respecting the De Rham diagram. For the pyramids, $(12 ; 13)$ and Zaglamayr cited in (16) have constructed approximation spaces or ordre $r$ respecting the exact sequence.

We now check that the approximation spaces constructed in this paper and in $(1 ; 2)$ respect the sequence (6).

Theorem 6. For any order $r$, the following sequence is exact

$$
\begin{aligned}
& \operatorname{Im} \operatorname{grad} P_{r}^{H^{1}}=\operatorname{Ker} P_{r}^{H(\text { curl })}=\left\{u \in P_{r}^{H(\text { curl })} \mid \text { curl } u=0\right\} \\
& \operatorname{Im} \operatorname{curl} P_{r}^{H(\text { curl })}=\operatorname{Ker} P_{r}^{H(d i v)}=\left\{u \in P_{r}^{H(d i v)} \mid \operatorname{div} u=0\right\}
\end{aligned}
$$




$$
\operatorname{Im} \operatorname{div} P_{r}^{H(d i v)}=\operatorname{Ker} P_{r}^{L^{2}}=P_{r}^{L^{2}}
$$

with the following spaces

$$
\begin{aligned}
& \hat{P}_{r}^{H^{1}}=\left\{\begin{array}{l}
\mathbb{P}_{r}(\hat{x}, \hat{y}, \hat{z}) \text { for the tetrahedron } \\
\mathbb{Q}_{r, r, r}(\hat{x}, \hat{y}, \hat{z}) \text { for the hexahedron } \\
\mathbb{W}_{r, r}(\hat{x}, \hat{y}, \hat{z}) \text { for the prism } \\
\mathbb{B}_{r}(\hat{x}, \hat{y}, \hat{z}) \text { for the pyramid }
\end{array}\right. \\
& \hat{P}_{r}^{H(c u r l)}=\left\{\begin{array}{l}
\mathcal{R}_{r}(\hat{x}, \hat{y}, \hat{z}) \text { for the tetrahedron } \\
\mathbb{Q}_{r-1, r+1, r+1}(\hat{x}, \hat{y}, \hat{z}) \times \mathbb{Q}_{r+1, r-1, r+1}(\hat{x}, \hat{y}, \hat{z}) \times \mathbb{Q}_{r+1, r+1, r-1}(\hat{x}, \hat{y}, \hat{z}) \text { for the hexahedron } \\
\left(\mathcal{R}_{r}(\hat{x}, \hat{y}) \otimes \mathbb{P}_{r+1}(\hat{z})\right) \times\left(\mathbb{P}_{r+1}(\hat{x}, \hat{y}) \otimes \mathbb{P}_{r-1}(\hat{z})\right) \text { for the triangular prism } \\
\mathcal{B}_{r}(\hat{x}, \hat{y}, \hat{z}) \text { for the pyramid }
\end{array}\right. \\
& \hat{P}_{r}^{H(d i v)}=\hat{P}_{r}^{s-o p t} \\
& \hat{P}_{r}^{L^{2}}=\left\{\begin{array}{l}
\mathbb{P}_{r-1}(\hat{x}, \hat{y}, \hat{z}) \text { for the tetrahedron } \\
\mathbb{Q}_{r, r, r}(\hat{x}, \hat{y}, \hat{z}) \oplus \hat{x}^{r+1} \mathbb{Q}_{r, r}(\hat{y}, \hat{z}) \oplus \hat{y}^{r+1} \mathbb{Q}_{r, r}(\hat{x}, \hat{z}) \oplus \hat{z}^{r+1} \mathbb{Q}_{r, r}(\hat{x}, \hat{y}) \oplus \text { for the hexahedron } \\
\mathbb{W}_{r, r}(\hat{x}, \hat{y}, \hat{z}) \oplus \mathbb{P}_{r-1}(\hat{x}, \hat{y}) \hat{z}^{r+1} \text { for the prism } \\
\left\{\begin{array}{ll}
\hat{x}^{i} \hat{y}^{j} & 0 \leq i, j \leq k+1 \\
(1-\hat{z})^{i+j-k}, & -1 \leq k \leq r-1
\end{array}\right\} \text { for the pyramid }
\end{array}\right.
\end{aligned}
$$

Proof. The proof is completed for the hexahedron. Let us take a function $u$ in $\mathbb{Q}_{r, r, r}$, obviously its gradient will belong to $\mathbb{Q}_{r-1, r, r} \times \mathbb{Q}_{r, r-1, r} \times \mathbb{Q}_{r, r, r-1}$, which is the space $P_{r}^{H(c u r l)}$. Therefore we have the inclusion

$$
\operatorname{Im} \operatorname{grad} P_{r}^{H^{1}} \subset \operatorname{Ker} P_{r}^{H(c u r l)} .
$$

Let us now take a function $u$ in $P_{r}^{H(c u r l)}$ such that $c u r l u=0$. Because of polynomial identification, it is equivalent to consider a function of the following form:

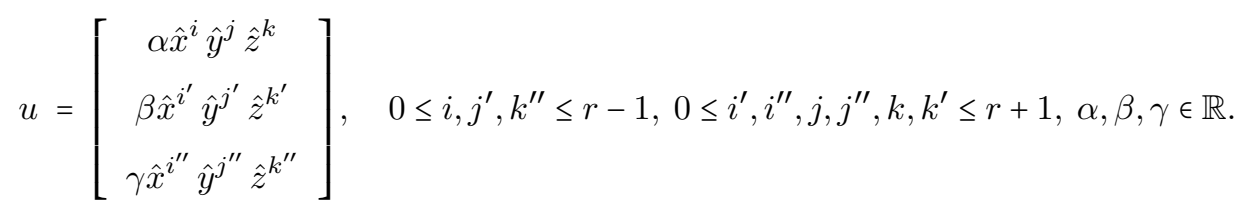

The curl of this function is equal to:

$$
\hat{\nabla} \times u=\left[\begin{array}{c}
\gamma j^{\prime \prime} \hat{x}^{i^{\prime \prime}} \hat{y}^{j^{\prime \prime}-1} \hat{z}^{k^{\prime \prime}}-\beta k^{\prime} \hat{x}^{i^{\prime}} \hat{y}^{j^{\prime}} \hat{z}^{k^{\prime}-1} \\
\alpha k \hat{x}^{i} \hat{y}^{j} \hat{z}^{k-1}-\gamma i^{\prime \prime} \hat{x}^{i^{\prime \prime}-1} \hat{y}^{j^{\prime \prime}} \hat{z}^{k^{\prime \prime}} \\
\beta i^{\prime} \hat{x}^{i^{\prime}-1} \hat{y}^{j^{\prime}} \hat{z}^{k^{\prime}}-\alpha j \hat{x}^{i} \hat{y}^{j-1} \hat{z}^{k}
\end{array}\right]
$$

Let us consider the case where $i^{\prime}, i^{\prime \prime}, j, j^{\prime \prime}, k, k^{\prime} \geq 1$, then $\alpha, \beta, \gamma \neq 0$ otherwise $u$ would be identically null. We obtain the following conditions in order to satisfy curl $u=0$ :

$$
\left\{\begin{array}{l}
a=i^{\prime \prime}=i^{\prime}=i+1 \\
b=j^{\prime \prime}=j=j^{\prime}+1 \\
c=k^{\prime}=k=k^{\prime \prime}+1
\end{array}\right.
$$


with $1 \leq a, b, c \leq r$ since $0 \leq i, j^{\prime}, k^{\prime \prime} \leq r-1 . \alpha, \beta, \gamma$ are solution of the linear system:

$$
\left\{\begin{array}{l}
\gamma b-\beta c=0 \\
\alpha c-\gamma a=0 \\
\beta a-\alpha b=0
\end{array} .\right.
$$

The rank of this system is equal to 2 , a non-trivial solution is equal to

$$
(\alpha, \beta, \gamma)=(a, b, c)
$$

Hence, we have obtained:

$$
u=\left[\begin{array}{c}
a \hat{x}^{a-1} \hat{y}^{b} \hat{z}^{c} \\
b \hat{x}^{a} \hat{y}^{b-1} \hat{z}^{c} \\
c \hat{x}^{a} \hat{y}^{b} \hat{z}^{c-1}
\end{array}\right]=\hat{\nabla}\left(\hat{x}^{a} \hat{y}^{b} \hat{z}^{c}\right)
$$

Since $1 \leq a, b, c \leq r, \hat{x}^{a} \hat{y}^{b} \hat{z}^{c} \in P_{r}^{H^{1}}$. Other conditions on $i, i^{\prime \prime}, j, j^{\prime \prime}, k, k^{\prime}$ lead to the same kind of result, with $0 \leq a, b, c \leq r$. Therefore we have proved that

$$
\operatorname{Ker} P_{r}^{H(c u r l)} \subset \operatorname{Im} \operatorname{grad} P_{r}^{H^{1}} .
$$

Since we have the two inclusions, these two spaces are equal. Let us now study the $H(d i v)$ space, another way to write the space for the hexahedron is the following one:

$$
\begin{aligned}
P_{r}^{H(d i v)} & =\left\{\left[\begin{array}{c}
\hat{x}^{i} \hat{y}^{j} \hat{z}^{k} \\
0 \\
0
\end{array}\right],\left[\begin{array}{c}
0 \\
\hat{x}^{j} \hat{y}^{i} \hat{z}^{k} \\
0
\end{array}\right],\left[\begin{array}{c}
0 \\
0 \\
\hat{x}^{j} \hat{y}^{k} \hat{z}^{i}
\end{array}\right], 0 \leq i \leq r+1,0 \leq j, k \leq r, j+k \neq 2 r\right\} \\
& \oplus\left\{\left[\begin{array}{c}
\hat{x}^{r+2} \hat{y}^{j} \hat{z}^{k} \\
0 \\
0
\end{array}\right],\left[\begin{array}{c}
0 \\
\hat{x}^{j} \hat{y}^{r+2} \hat{z}^{k} \\
0
\end{array}\right],\left[\begin{array}{c}
0 \\
0 \\
\hat{x}^{j} \hat{y}^{k} \hat{z}^{r+2}
\end{array}\right], 0 \leq j, k \leq r\right\} \\
& \oplus\left\{\left[\begin{array}{c}
\hat{x}^{r+1} \hat{y}^{r} \hat{z}^{r} \\
\hat{x}^{r} \hat{y}^{r+1} \hat{z}^{r} \\
\hat{x}^{r} \hat{y}^{r} \hat{z}^{r+1}
\end{array}\right]\right\}
\end{aligned}
$$

The two last groups have clearly no intersection with Ker $d i v$, it is not possible to construct a function $u$ as a combination of the basis functions of the two last groups and ensure div $u=0$. Therefore, we will restrict our interest to the first group. For this first group, the condition is that $j, k$ are lower than $r$ but cannot be both equal to $r$. Let us consider $u$ in $P_{r}^{H(\text { curl })}$, we have already computed the curl of such a representative function. The $x$-component of the curl is equal to

$$
\gamma j^{\prime \prime} \hat{x}^{i^{\prime \prime}} \hat{y}^{j^{\prime \prime}-1} \hat{z}^{k^{\prime \prime}}-\beta k^{\prime} \hat{x}^{i^{\prime}} \hat{y}^{j^{\prime}} \hat{z}^{k^{\prime}-1}
$$

The first polynomial is such that:

$$
\operatorname{deg} \hat{x}=i^{\prime \prime} \leq r+1, \quad \operatorname{deg} \hat{y}=j^{\prime \prime}-1 \leq r, \quad \operatorname{deg} \hat{y}=k^{\prime \prime} \leq r-1
$$

and the second polynomial

$$
\operatorname{deg} \hat{x}=i^{\prime} \leq r+1, \quad \operatorname{deg} \hat{y}=j^{\prime} \leq r-1, \quad \operatorname{deg} \hat{z}=k^{\prime}-1 \leq r .
$$

These polynomial are indeed belonging to $P_{r}^{H(d i v)}$, since the degrees in y and $\mathrm{z}$ cannot be both equal to $r$. Similar observations on $y$ and $z$-components of the curl lead us to the following inclusion

$$
\operatorname{Im} \text { curl } P_{r}^{H(c u r l)} \subset \operatorname{Ker} P_{r}^{H(d i v)} \text {. }
$$


As for $H($ curl $)$, we take a function $u$ of the form:

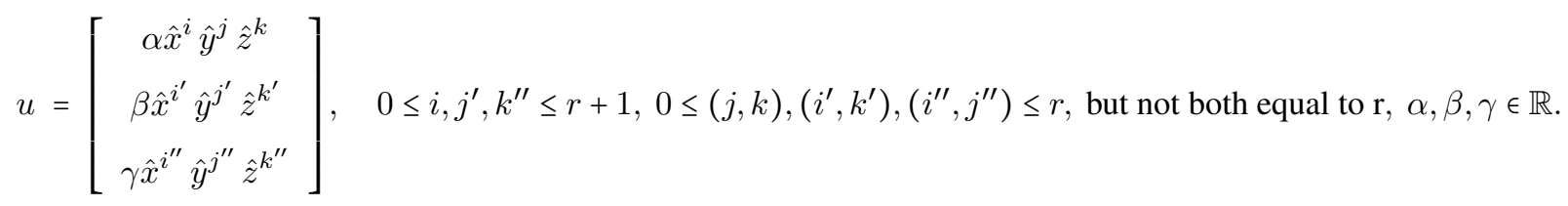

The divergence of $u$ is equal to:

$$
\operatorname{div} u=\alpha i \hat{x}^{i-1} \hat{y}^{j} \hat{z}^{k}+\beta j^{\prime} \hat{x}^{i^{\prime}} \hat{y}^{j^{\prime}-1} \hat{z}^{k^{\prime}}+\gamma k^{\prime \prime} \hat{x}^{i^{\prime \prime}} \hat{y}^{j^{\prime \prime}} \hat{z}^{k^{\prime \prime}-1} .
$$

Let us consider that $i, j^{\prime}, k^{\prime \prime} \geq 1$, then we obtain

$$
\left\{\begin{array}{l}
a-1=i^{\prime}=i^{\prime \prime}=i-1 \\
b-1=j=j^{\prime \prime}=j^{\prime}-1 \\
c-1=k=k^{\prime}=k^{\prime}-1
\end{array}\right.
$$

$u$ is equal to

$$
u=\left[\begin{array}{c}
\alpha \hat{x}^{a} \hat{y}^{b-1} \hat{z}^{c-1} \\
\beta \hat{x}^{a-1} \hat{y}^{b} \hat{z}^{c-1} \\
\gamma \hat{x}^{a-1} \hat{y}^{b-1} \hat{z}^{c}
\end{array}\right]
$$

with the condition

$$
\alpha a+\beta b+\gamma c=0
$$

A set of non-trivial solutions is equal to

$$
\left[\begin{array}{c}
\alpha \\
\beta \\
\gamma
\end{array}\right]=\left[\begin{array}{c}
b \\
-a \\
0
\end{array}\right] \text {, or }\left[\begin{array}{c}
c \\
0 \\
-a
\end{array}\right] \text {, or }\left[\begin{array}{c}
0 \\
c \\
-b
\end{array}\right]
$$

Only two functions among these three are needed to generate all the solutions. Let us consider the first non-trivial solution, $u$ is then equal to:

$$
u=\left[\begin{array}{c}
b \hat{x}^{a} \hat{y}^{b-1} \hat{z}^{c-1} \\
-a \hat{x}^{a-1} \hat{y}^{b} \hat{z}^{c-1} \\
0
\end{array}\right]=\operatorname{curl}\left[\begin{array}{c}
0 \\
0 \\
\hat{x}^{a} \hat{y}^{b} \hat{z}^{c-1}
\end{array}\right] .
$$

If $u$ belongs to the first group of $P_{r}^{H(d i v)}$, we have the following conditions

$$
a, b, c \leq r+1 \text {. }
$$

However if $c=r+1$, we have $a, b \leq r$, since $a-1, c-1$ or $a-1, b-1$ can not be both equal to $r$. But in that case, we are writing $u$ as the curl of the following function

$$
\left[\begin{array}{c}
b \hat{x}^{a} \hat{y}^{b-1} \hat{z}^{c-1} \\
-a \hat{x}^{a-1} \hat{y}^{b} \hat{z}^{c-1} \\
0
\end{array}\right]=\operatorname{curl}\left[\begin{array}{c}
-a \hat{x}^{a-1} \hat{y}^{b} \hat{z}^{c-1} \\
-b \hat{x}^{a} \hat{y}^{b-1} \hat{z}^{c-1} \\
0
\end{array}\right]
$$

This last function belongs to $P_{r}^{H(c u r l)}$ since $a, b \leq r$. The two other sets of $(\alpha, \beta, \gamma)$ can be treated in a symmetric manner.

Eventually, we have proved the inclusion

$$
\operatorname{Ker} P_{r}^{H(d i v)} \subset \operatorname{Im} \operatorname{curl} P_{r}^{H(c u r l)}
$$

Since we have the two inclusions, these two spaces are equal. 
Remark 4. It should be noticed that the sequence is exact for super-optimal spaces $\hat{P}_{r}^{H(d i v)}=\hat{P}_{r}^{s-o p t}$. This exactness does not hold if we use optimal spaces $\hat{P}_{r}^{H(d i v)}=\hat{P}_{r}^{o p t}$. In that last case, we have only inclusions and not equalities. We have observed that:

\section{- Hexahedron:}

$$
\operatorname{dim} \operatorname{Ker} \hat{P}_{r}^{o p t}=\operatorname{dim} \operatorname{Im} \operatorname{curl} P_{r}^{H(c u r l)}+(3 r+5)
$$

- Triangular prism:

$$
\operatorname{dim} \operatorname{Ker} \hat{P}_{r}^{o p t}=\operatorname{dim} \operatorname{Im} \operatorname{curl} P_{r}^{H(c u r l)}+(r+2)
$$

Therefore the additional functions of the optimal spaces (compared to super-optimal spaces) are only contributing to the kernel of the divergence.

\section{Numerical Integration}

The stiffness matrix of the variational formulation (2) reads (when $\rho=1$ )

$$
K_{i j}=\int_{\Omega} \operatorname{div} \varphi_{i} \operatorname{div} \varphi_{j} d x=\sum_{K} \int_{K} \operatorname{div} \varphi_{i} \operatorname{div} \varphi_{j} d x
$$

where

$$
\int_{K} \operatorname{div} \varphi_{i} \operatorname{div} \varphi_{j} d x=\int_{\hat{K}} \frac{1}{|D F|} \operatorname{div} \hat{\varphi}_{i} \operatorname{div} \hat{\varphi}_{j} d \hat{x}
$$

Because of the presence of $\frac{1}{|D F|}$, this integral can not be computed exactly by using numerical integration based on Gauss points. This issue is also present for the mass matrix, since

$$
\int_{K} \varphi_{i} \cdot \varphi_{j} d x=\int_{\hat{K}} \frac{1}{|D F|} D F^{*} D F \hat{\varphi}_{i} \cdot \hat{\varphi}_{j} d x .
$$

However, one can expect as for continuous elements (see (1)) that the use of numerical integration does not deteriorate the order of convergence.

The main ingredients of the numerical integration consists of Gauss and Gauss-Jacobi rules, which are quadrature formulas exact for respectively $\mathbb{P}_{2 r+1}(x)$ and $(1-x)^{\alpha}(1+x)^{\beta} \mathbb{P}_{2 r+1}(x)$, where we take $\alpha=1, \beta=0$. In the sequel, we will not specify if the Gauss formulas are adapted to the interval $[0,1]$ or to the interval $[-1,1]$, the interval for each coordinate will depend on the reference element $\hat{K}$. We also use symmetric rules adapted for the integration over the unit triangle and unit tetrahedron (see (18) for a brief review of these formulas).

Conjecture 1. The error of convergence $\left\|u-u_{h}\right\|_{H(d i v, \Omega)}$ of the exact solution $u$ of (1) toward the solution $u_{h}$ of the discrete variational formulation (4) with optimal finite element spaces $\hat{P}_{r}^{\text {opt }}(\hat{K})$ is in $O\left(h^{r}\right)$ when the integrals are computed numerically on each element $\hat{K}$ with the following rules:

- Tetrahedron: A quadrature rule $\left(\omega_{k}^{\text {tet }}, \xi_{k}^{\text {tet }}\right)$ exact for the integration of $\mathbb{P}_{2 r}(\hat{K})$

- Hexahedron: A quadrature rule $\left(\omega_{k_{1}, k_{2}, k_{3}}, \xi_{k_{1}, k_{2}, k_{3}}\right)$ exact for the integration of $\mathbb{Q}_{2 r+5}(\hat{K})$, with Gauss points:

$$
\omega_{k_{1}, k_{2}, k_{3}}=\omega_{k_{1}}^{G} \omega_{k_{2}}^{G} \omega_{k_{3}}^{G}, \quad \xi_{k_{1}, k_{2}, k_{3}}=\left(\xi_{k_{1}}^{G}, \xi_{k_{2}}^{G}, \xi_{k_{3}}^{G}\right), \quad 0 \leq k_{1}, k_{2}, k_{3} \leq r+2
$$

with $r+3$ Gauss points $\left(\omega_{k_{1}}^{G}, \xi_{k_{1}}^{G}\right)$

- Prism: A quadrature rule $\left(\omega_{k_{1}, k_{3}}, \xi_{k_{1}, k_{3}}\right)$ exact for the integration of $\mathbb{P}_{2 r+2}(\hat{x}, \hat{y}) \times \mathbb{P}_{2 r+5}(\hat{z})$, with the following tensor structure:

$$
\omega_{k_{1}, k_{3}}=\omega_{k_{1}}^{t r i} \omega_{k_{3}}^{G}, \quad \xi_{k_{1}, k_{3}}=\left(\xi_{k_{1}}^{t r i}, \xi_{k_{3}}^{G}\right)
$$

with $r+3$ Gauss points $\left(\omega_{k_{3}}^{G}, \xi_{k_{3}}^{G}\right)$ and a quadrature rule adapted to triangles $\left(\omega_{k_{1}}^{\text {tri }}, \xi_{k_{1}}^{\text {tri }}\right)$ 
- Pyramid: A quadrature rule $\left(\omega_{k_{1}, k_{2}, k_{3}}, \xi_{k_{1}, k_{2}, k_{3}}\right)$ exact for the integration of $(1-\widetilde{z}) \mathbb{Q}_{2 r+3}(\widetilde{x}, \widetilde{y}, \widetilde{z})$ with the following tensor structure:

$$
\omega_{k_{1}, k_{2}, k_{3}}=\left(1-\xi_{k_{3}}^{G}\right) \omega_{k_{1}}^{G} \omega_{k_{2}}^{G} \omega_{k_{3}}^{G J}, \quad \xi_{k_{1}, k_{2}, k_{3}}=\left(\left(1-\xi_{k_{3}}^{G}\right) \xi_{k_{1}}^{G},\left(1-\xi_{k_{3}}^{G}\right) \xi_{k_{2}}^{G}, \xi_{k_{3}}^{G J}\right)
$$

with $r+2$ Gauss points $\left(\omega_{k_{1}}^{G}, \xi_{k_{1}}^{G}\right)$ and Gauss-Jacobi points $\left(\omega_{k_{3}}^{G J}, \xi_{k_{3}}^{G J}\right)$ exact for the integration of $(1-$ z) $\mathbb{P}_{2 r+3}(z)$.

Remark 5. Unlike the other elements, the quadrature rule used for the pyramid is actually not exact when the element is affine (DF being constant) because the divergence of $\hat{P}_{r}^{\text {opt }}$ contains the singular function $\frac{1}{1-z}$. To get an exact integration for affine pyramids, Gauss quadrature rules should be preferred to Gauss-Jacobi rules:

$$
\omega_{k_{1}, k_{2}, k_{3}}=\left(1-\xi_{k_{3}}^{G}\right)^{2} \omega_{k_{1}}^{G} \omega_{k_{2}}^{G} \omega_{k_{3}}^{G}, \quad \xi_{k_{1}, k_{2}, k_{3}}=\left(\left(1-\xi_{k_{3}}^{G}\right) \xi_{k_{1}}^{G},\left(1-\xi_{k_{3}}^{G}\right) \xi_{k_{2}}^{G}, \xi_{k_{3}}^{G}\right) .
$$

However with the quadrature rules using Gauss-Jacobi, we obtain numerically the expected order of convergence whereas we obtain a non-consistent method for any order of approximation when using Gauss-Jacobi rules $\left(\omega_{k_{3}}^{G J, 2}, \xi_{k_{3}}^{G J, 2}\right)$ exact for the integration of $(1-z)^{2} \mathbb{P}_{2 r+3}(z)$, that is the following quadrature rules:

$$
\omega_{k_{1}, k_{2}, k_{3}}=\omega_{k_{1}}^{G} \omega_{k_{2}}^{G} \omega_{k_{3}}^{G J, 2}, \quad \xi_{k_{1}, k_{2}, k_{3}}=\left(\left(1-\xi_{k_{3}}^{G}\right) \xi_{k_{1}}^{G},\left(1-\xi_{k_{3}}^{G}\right) \xi_{k_{2}}^{G}, \xi_{k_{3}}^{G J, 2}\right)
$$

This is a specificity of $H($ div $)$ space, since such a rule works very well for $H^{1}(\Omega)($ see $(1))$ and $H($ curl, $\Omega)(q u a d r a-$ ture formula used to obtain the results in (2)).

\section{First Family}

Finite element spaces of Nedelec's first family are denoted $\hat{P}_{r}^{1}$ and equal (see (15)) to

- Tetrahedron:

$$
\hat{P}_{r}^{1}=\mathfrak{D}_{r}(\hat{x}, \hat{y}, \hat{z})
$$

- Hexahedron:

$$
\hat{P}_{r}^{1}=\mathbb{Q}_{r, r-1, r-1}(\hat{x}, \hat{y}, \hat{z}) \times \mathbb{Q}_{r-1, r, r-1}(\hat{x}, \hat{y}, \hat{z}) \times \mathbb{Q}_{r-1, r-1, r}(\hat{x}, \hat{y}, \hat{z})
$$

- Triangular prism:

$$
\hat{P}_{r}^{1}=\mathfrak{D}_{r}(\hat{x}, \hat{y}) \mathbb{P}_{r-1}(\hat{z}) \times \mathbb{P}_{r-1}(\hat{x}, \hat{y}) \mathbb{P}_{r}(\hat{z})
$$

- Pyramid:

$$
\begin{aligned}
\hat{P}_{r}^{1}= & \mathbb{B}_{r-1}(\hat{x}, \hat{y}, \hat{z})^{3} \\
& \left.\oplus\left[\begin{array}{c}
\frac{\hat{x}^{n+1} \hat{y}^{m}}{(1-\hat{z})^{m+1}} \\
0 \\
0
\end{array}\right] \oplus\left[\begin{array}{c}
0 \\
\frac{\hat{x}^{m} \hat{y}^{n+1}}{(1-\hat{z})^{m+1}} \\
0
\end{array}\right] 0 \leq m \leq n \leq r-1\right\} \\
& \oplus\left\{\begin{array}{c}
\left.\frac{\hat{x}^{m} \hat{y}^{n+1}}{(1-\hat{z})^{m+1}}\left[\begin{array}{c}
\frac{\hat{x}}{(1-\hat{z})} \\
0 \\
-1
\end{array}\right] \oplus \frac{\hat{x}^{n+1} \hat{y}^{m}}{(1-\hat{z})^{m+1}}\left[\begin{array}{c}
0 \\
\frac{\hat{y}}{(1-\hat{z})} \\
-1
\end{array}\right] 0 \leq m \leq n \leq r-2\right\} \\
\oplus \frac{\hat{x}}{(1-\hat{z})^{i+j-r}}\left[\begin{array}{c}
(1-\hat{z}) \\
\frac{\hat{y}}{(1-\hat{z})} \\
-1
\end{array}\right], 0 \leq i, j \leq r-1 \\
\end{array}\right.
\end{aligned}
$$


There is no agreement about the finite element space for pyramids, the proposed space is a slight modification of the optimal space such that the degrees of functions involved in $\hat{P}_{r}^{1}$ are lower or equal to $r$.

Remark 6. The normal traces of classical finite element spaces $\hat{P}_{r}^{1}$ are equal to

$$
\begin{gathered}
\mathbb{Q}_{r-1, r-1}(x, y) \text { for quadrilateral faces }, \\
\mathbb{P}_{r-1}(x, y) \text { for triangular faces } .
\end{gathered}
$$

We remark that the spaces $\hat{P}_{r+1}^{1}$ and $\hat{P}_{r}^{\text {opt }}$ have the same number of degrees offreedom on quadrilateral faces, therefore these two spaces will be often compared in the numerical results.

\section{Hierarchical Functions}

We present here simple hierarchical basis for optimal finite element spaces $\hat{P}_{r}^{o p t}$ by using Jacobi polynomials $P_{i, j}^{\alpha, \beta}$, but other choices could be considered.

Proposition 2. The following basis functions are an hierarchical basis $H$ (div) conforming of $\hat{P}_{r}^{o p t}$

\section{- Hexahedron:}

\section{HIERARCHICAL H(DIV) FUNCTIONS FOR THE CUBE}

Additional conditions and functions to get a hierarchical base of $\hat{P}_{r}^{s-o p t}$ are indicated in red.

For the faces:

$$
\begin{aligned}
& {\left[\begin{array}{c}
\hat{x} P_{i}^{0,0}(2 \hat{y}-1) P_{j}^{0,0}(2 \hat{z}-1) \\
0 \\
0
\end{array}\right], \quad\left[\begin{array}{c}
(1-\hat{x}) P_{i}^{0,0}(2 \hat{y}-1) P_{j}^{0,0}(2 \hat{z}-1) \\
0 \\
0
\end{array}\right], \quad 0 \leq i, j \leq r, i+j \neq 2 r} \\
& {\left[\begin{array}{c}
0 \\
\hat{y} P_{i}^{0,0}(2 \hat{x}-1) P_{j}^{0,0}(2 \hat{z}-1) \\
0
\end{array}\right], \quad\left[\begin{array}{c}
(1-\hat{y}) P_{i}^{0,0}\left(\begin{array}{c}
0 \\
0 \\
0
\end{array}\right) P_{j}^{0,0}(2 \hat{z}-1) \\
0
\end{array}\right], \quad 0 \leq i, j \leq r, i+j \neq 2 r} \\
& {\left[\begin{array}{c}
0 \\
0 \\
\hat{z} P_{i}^{0,0}(2 \hat{x}-1) P_{j}^{0,0}(2 \hat{y}-1)
\end{array}\right], \quad\left[\begin{array}{c}
0 \\
0 \\
(1-\hat{z}) P_{i}^{0,0}(2 \hat{x}-1) P_{j}^{0,0}(2 \hat{y}-1)
\end{array}\right], \quad 0 \leq i, j \leq r, i+j \neq 2 r}
\end{aligned}
$$

For the interior functions :

$$
\begin{aligned}
& {\left[\begin{array}{c}
\hat{x}(1-\hat{x}) P_{i}^{1,1}(2 \hat{x}-1) P_{j}^{0,0}(2 \hat{y}-1) P_{k}^{0,0}(2 \hat{z}-1) \\
0 \\
0
\end{array}\right], \quad 0 \leq i, j, k \leq r, \quad i=r \text { or } j \neq r \text { or } k \neq r} \\
& {\left[\begin{array}{c}
0 \\
\hat{y}(1-\hat{y}) P_{i}^{1,1}(2 \hat{y}-1) P_{j}^{0,0}(2 \hat{x}-1) P_{k}^{0,0}(2 \hat{z}-1) \\
0
\end{array}\right], \quad 0 \leq i, j, k \leq r, \quad i=r \text { or } j \neq r \text { or } k \neq r} \\
& 0 \\
& 0 \\
& {\left[\begin{array}{c}
\hat{z}(1-\hat{z}) P_{i}^{1,1}(2 \hat{z}-1) P_{j}^{0,0}(2 \hat{x}-1) P_{k}^{0,0}(2 \hat{y}-1)
\end{array}\right], \quad 0 \leq i, j, k \leq r, \quad i=r \text { or } j \neq \text { ror } k \neq r}
\end{aligned}
$$

Linked function

$$
\left[\begin{array}{c}
\hat{x}(1-\hat{x}) P_{r-1}^{1,1}(2 \hat{x}-1) P_{r}^{0,0}(2 \hat{y}-1) P_{r}^{0,0}(2 \hat{z}-1) \\
\hat{y}(1-\hat{y}) P_{r-1}^{1,1}(2 \hat{y}-1) P_{r}^{0,0}(2 \hat{x}-1) P_{r}^{0,0}(2 \hat{z}-1) \\
\hat{z}(1-\hat{z}) P_{r-1}^{1,1}(2 \hat{z}-1) P_{r}^{0,0}(2 \hat{x}-1) P_{r}^{0,0}(2 \hat{y}-1)
\end{array}\right]
$$




\section{- Triangular prism:}

\section{HIERARCHICAL H(DIV) FUNCTIONS FOR THE PRISM}

For the faces :

$$
\begin{aligned}
& P_{i}^{0,0}\left(\frac{2 \hat{x}}{1-\hat{y}}-1\right)(1-\hat{y})^{i} P_{j}^{2 i+1,0}(2 \hat{y}-1)\left[\begin{array}{c}
0 \\
0 \\
1-\hat{z}
\end{array}\right], \quad 0 \leq i+j \leq r-1 \\
& P_{i}^{0,0}\left(\frac{2 \hat{x}}{1-\hat{y}}-1\right)(1-\hat{y})^{i} P_{j}^{2 i+1,0}(2 \hat{y}-1)\left[\begin{array}{c}
0 \\
0 \\
\hat{z}
\end{array}\right], \quad 0 \leq i+j \leq r-1 \\
& P_{i}^{0,0}(2 \hat{x}-1) P_{j}^{0,0}(2 \hat{z}-1)\left[\begin{array}{c}
\hat{x} \\
\hat{y}-1 \\
0
\end{array}\right], \quad 0 \leq i, j \leq r \\
& P_{i}^{0,0}(2 \hat{y}-1) P_{j}^{0,0}(2 \hat{z}-1)\left[\begin{array}{c}
\hat{x} \\
\hat{y} \\
0
\end{array}\right], \quad 0 \leq i, j \leq r \\
& P_{i}^{0,0}(2 \hat{y}-1) P_{j}^{0,0}(2 \hat{z}-1)\left[\begin{array}{c}
-(\hat{x}-1) \\
-\hat{y} \\
0
\end{array}\right], \quad 0 \leq i, j \leq r
\end{aligned}
$$

For the interior functions :

$$
\begin{aligned}
& P_{i, j, k}\left[\begin{array}{c}
\hat{x} \hat{y} \\
\hat{y}(\hat{y}-1) \\
0
\end{array}\right], \quad P_{i, j, k}\left[\begin{array}{c}
\hat{x}(\hat{x}-1) \\
\hat{x} \hat{y} \\
0
\end{array}\right], \quad P_{i, j, k}\left[\begin{array}{c}
0 \\
0 \\
\hat{z}(1-\hat{z})
\end{array}\right], \\
& \text { with } P_{i, j, k}=P_{i}^{0,0}\left(\frac{2 \hat{x}}{1-\hat{y}}-1\right)(1-\hat{y})^{i} P_{j}^{2 i+1,0}(2 \hat{y}-1) P_{k}^{0,0}(2 \hat{z}-1) \\
& \qquad i, j, k \geq 0, i+j \leq r-1, k \leq r
\end{aligned}
$$




\section{- Pyramid:}

\section{HIERARCHICAL H(DIV) FUNCTIONS FOR THE PYRAMID}

Conditions to get a hierarchical base of $\hat{P}_{r}^{1}$ are indicated in blue.

For the faces :

$$
\begin{aligned}
& \frac{1}{4} P_{i}^{0,0}\left(\frac{\hat{x}}{1-\hat{z}}\right) P_{j}^{0,0}\left(\frac{\hat{y}}{1-\hat{z}}\right)(1-\hat{z})^{\max (i, j)-1}\left[\begin{array}{c}
-\hat{x} \\
-\hat{y} \\
1-\hat{z}
\end{array}\right], \quad 0 \leq i, j \leq r \\
& P_{i, j}^{1}\left[\begin{array}{c}
\frac{-\hat{x} \hat{z}}{1-\hat{z}} \\
\frac{-2(1-\hat{y}-\hat{z})-\hat{y} \hat{z}}{1-\hat{z}} \\
\hat{z}
\end{array}\right], \quad P_{i, j}^{1}\left[\begin{array}{c}
\frac{-\hat{x} \hat{z}}{1-\hat{z}} \\
\frac{2(1+\hat{y}-\hat{z})-\hat{y} \hat{z}}{1-\hat{z}} \\
\hat{z}
\end{array}\right] \\
& P_{i, j}^{2}\left[\begin{array}{c}
\frac{-2(1-\hat{x}-\hat{z})-\hat{x} \hat{z}}{1-\hat{z}} \\
\frac{-\hat{y} \hat{z}}{1-\hat{z}} \\
\hat{z}
\end{array}\right], \quad P_{i, j}^{1}\left[\begin{array}{c}
\frac{2(1+\hat{x}-\hat{z})-\hat{x} \hat{z}}{1-\hat{z}} \\
\frac{-\hat{y} \hat{z}}{1-\hat{z}} \\
\hat{z}
\end{array}\right] \\
& \text { with } P_{i, j}^{1}=\frac{1}{8} P_{i}^{0,0}\left(\frac{\hat{x}}{1-\hat{z}}\right)(1-\hat{z})^{i} P_{j}^{2 i+1,0}(2 \hat{z}-1) \text { and } P_{i, j}^{2}=\frac{1}{8} P_{i}^{0,0}\left(\frac{\hat{y}}{1-\hat{z}}\right)(1-\hat{z})^{i} P_{j}^{2 i+1,0}(2 \hat{z}-1) \\
& i+j \leq r-1
\end{aligned}
$$

For the interior functions :

$$
\begin{aligned}
& P_{i, j, k}\left[\begin{array}{c}
-\hat{x} \hat{z} \\
-\hat{y} \hat{z} \\
(1-\hat{z}) \hat{z}
\end{array}\right], \quad i, j, k \geq 0, i, j \leq r-1, k \leq r-1-\max (i, j) \\
& P_{i, j, k}\left[\begin{array}{c}
\frac{\hat{x} \hat{y} \hat{z}}{1-\hat{z}} \\
1-\hat{z}-\hat{y}^{2} \\
-\hat{y} \hat{z}
\end{array}\right], \begin{array}{l}
i, j, k \geq 0, i, j \leq r-1, k \leq r-1-\max (i, j) \\
i, j, k \geq 0, i \leq r-1, j \leq r-2, k \leq r-1-\max (i, j)
\end{array} \\
& P_{i, j, k}\left[\begin{array}{c}
1-\hat{z}-\hat{x}^{2} \\
\frac{\hat{x} \hat{y} \hat{z}}{1-\hat{z}} \\
-\hat{x} \hat{z}
\end{array}\right], \quad \begin{array}{l}
i, j, k \geq 0, i, j \leq r-1, k \leq r-1-\max (i, j) \\
i, j, k \geq 0, i \leq r-2, j \leq r-1, k \leq r-1-\max (i, j)
\end{array} \\
& \text { with } P_{i, j, k}=P_{i}^{0,0}\left(\frac{\hat{x}}{1-\hat{z}}\right) P_{j}^{0,0}\left(\frac{\hat{y}}{1-\hat{z}}\right)(1-\hat{z})^{\max (i, j)-1} P_{k}^{2 \max (i, j)+2,0}(2 \hat{z}-1)
\end{aligned}
$$

The hierarchic base for the first family for pyramids is constructed by changing the bounds for the indices 


\section{- Tetrahedron:}

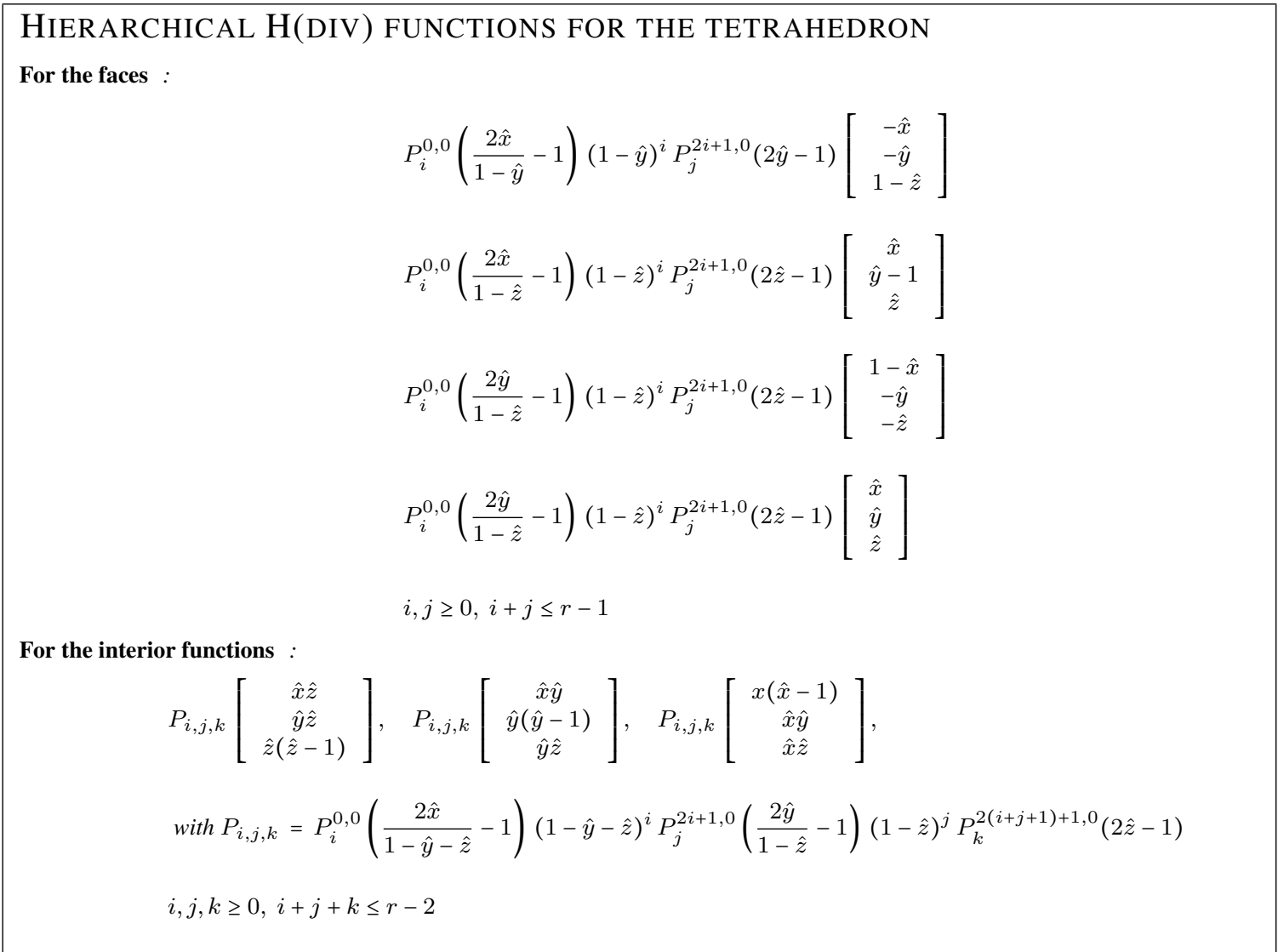

\section{Comparison between Elements}

\subsection{Pyramidal Elements}

\subsubsection{Theoretical Point of View}

We have compared our finite element spaces with the following spaces found in the literature. Below the remarks about this comparison on a theoretical point of view:

1. For $r=1$, the spaces proposed in (19), (14), (13) are the same, and almost coincide with the space $\hat{P}_{r}^{1} . \hat{P}_{r}^{1}$ contains six degrees of freedom: if the interior degree of freedom is removed, and the basis function associated with the quadrilateral face replaced by

$$
\left[\begin{array}{c}
\hat{x} \\
\hat{y} \\
-(1-\hat{z})
\end{array}\right]
$$

we obtain the coincidence with the "classical" space of the literature.

2. The proposed space $\hat{P}_{r}^{1}$ satisfies the following conditions:

$\left\{\begin{array}{l}\hat{P}_{r}^{1} \supset \mathbb{D}_{r} \Rightarrow \text { optimal estimate for affine pyramids } \\ \left.\hat{P}_{r}^{1} \supset \hat{P}_{r-1}^{o p t} \Rightarrow \text { convergence in } O\left(h^{r-1}\right)\right) \text { for non-affine pyramids } \\ \text { Degrees of basis functions in } \widetilde{x}, \widetilde{y}, \widetilde{z} \text { is lower or equal to } \mathrm{r} \Rightarrow \text { less expensive quadrature rules than for } \hat{P}_{r}^{\text {opt }}\end{array}\right.$

Therefore, we think that this space is quite attractive to use in conjunction with finite element space of the first kind for the other elements. 
3. Basis functions proposed in (14) for $r \geq 2$ rely on the multiplication of first-order basis functions with polynomials in $\hat{x}, \hat{y}, \hat{z}$, but as we know, polynomials are not suitable for the pyramid. As a result, Graglia's space $G_{2}$ of second-order have the following properties:

$$
\left\{\begin{array}{l}
G_{2} \subset \hat{P}_{3}^{o p t}, \text { but } G_{2} \not \subset \hat{P}_{2}^{o p t} \\
G_{2} \supset \mathbb{D}_{2} \\
G_{2} \not \hat{P}_{1}^{o p t} .
\end{array}\right.
$$

Therefore, these functions should lead to a convergence in $O\left(h^{2}\right)$ for affine pyramids, and be non-consistent for non-affine pyramids. Furthermore, as for $H(\mathrm{curl})$ approximation, we have also found the presence of spurious modes, mainly concentrated on the lower part of the spectrum.

4. Finite element space proposed in (12) $N P_{r}$ has the following properties:

$$
\left\{\begin{array}{l}
N P_{r} \not \hat{P}_{r}^{o p t} \\
N P_{r} \supset \mathbb{D}_{r} \\
N P_{r} \not \hat{P}_{1}^{o p t}
\end{array}\right.
$$

This space should give an optimal convergence in $O\left(h^{r}\right)$ for affine pyramids, but should be non-consistent for non-affine pyramids. Furthermore the number of degrees of freedom becomes very large when $r$ is high. In the numerical comparisons, this space will be called the first Nigam Phillips space. The basis functions proposed in the paper seem to be miswritten. The proposed representative function of a triangular function does not belong to the space, a correct expression of this function (with the notations of the paper) is equal to

$$
\frac{1}{(1+z)^{k+2}}\left[\begin{array}{c}
0 \\
2(1-y) x^{a} z^{b} \\
-x^{a} z^{b+1}
\end{array}\right], \quad a+b \leq k-1
$$

5. Finite element space proposed in (13) $N P_{r}$ is a nice attempt to reduce the number of degrees of freedom of the first space. This second space has the following properties

$$
\left\{\begin{array}{l}
N P_{r} \subset \hat{P}_{r}^{1} \\
N P_{r} \supset \mathbb{D}_{r} \\
N P_{r} \not \hat{P}_{1}^{o p t}
\end{array}\right.
$$

Hence, we think that this space should give an optimal convergence in $O\left(h^{r}\right)$ for affine pyramids, but should be non-consistent for non-affine pyramids. It should be noticed that the dimension of this space is smaller than the dimension of $\hat{P}_{r}^{1}$. This space seems attractive in the case where the mesh contains only affine pyramids.

\subsubsection{Dispersion Analysis}

A dispersion analysis (see (1) for more details) is performed, it consists of computing the numerical pulsation $\omega_{h}$ for a given wave vector $\mathbf{k}$. The value $\omega_{h}$ found should be close to the continuous dispersion relation:

$$
\omega=\|\mathbf{k}\|
$$

We call dispersion error the following ratio:

$$
\text { dispersion error }=\frac{\omega_{h}-\|\mathbf{k}\|}{\|\mathbf{k}\|} .
$$



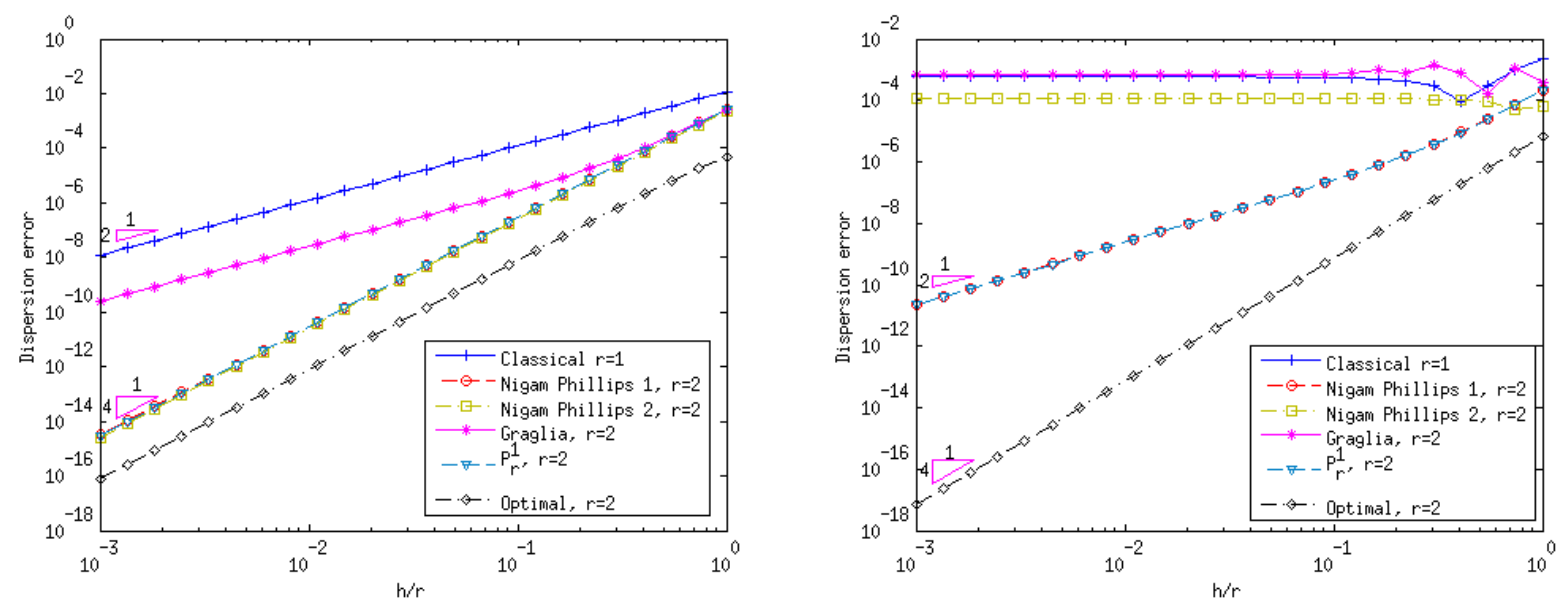

Figure 2: Dispersion error for different pyramidal spaces on a pattern containing only affine (left) and non-affine (right) pyramids.

The wave vector $\mathbf{k}$ is a $3-\mathrm{D}$ vector, the dispersion error depends on its orientation. For the sake of simplicity, we will always take this vector in the direction of $x$-coordinates:

$$
\mathbf{k}=\left[\begin{array}{c}
k \\
0 \\
0
\end{array}\right]
$$

The dispersion analysis is performed on a periodic pattern, it is therefore equivalent to decrease $k$ and take a mesh size $h=1$ or decrease $h$ and take a wave vector $k=1$. This is the last case that will be chosen, and the dispersion error will be displayed versus the mesh size.

The dispersion analysis is performed for affine pyramids, by using a regular pattern (see Fig. 4) where each cube is split into six pyramids. The dispersion error found for the different finite elements is represented in Fig. 2 . It is observed that Graglia's basis functions provide a dispersion error in $O\left(h^{2}\right)$ (for $r=2$ ), whereas other spaces are providing a dispersion error in $O\left(h^{2 r}\right)$. Nigam \& Phillips spaces and $\hat{P}_{r}^{1}$ have almost the same dispersion error, whereas the optimal finite space seems less dispersive.

The same analysis is conducted with a deformed pattern (see Fig. 4) containing both affine and non-affine pyramids, in Fig. 2. It is observed that the classical first order functions, Graglia's basis functions (for $r=2$ ) and second Nigam \& Phillips space are providing a dispersion error in $O(1)$ (non-consistent), whereas first Nigam \& Phillips space and $\hat{P}_{r}^{1}$ give almost the same dispersion error in $O\left(h^{2 r-2}\right)$, and optimal elements are the less dispersive elements with a convergence in $O\left(h^{2 r}\right)$. These assumptions have also been numerically checked for $r=3$. It should be noticed that the consistency error of second Nigam \& Phillips space is much smaller when $r=2$ than when $r=1$, and it decreases furthermore for high orders. Therefore, we think that this space is not $h$-convergent but probably $p$-convergent for non-affine pyramids.

\subsubsection{Convergence for the Source Problem}

Next, the convergence in $H$ (div) norm is studied for the source problem (1) with $f$ a gaussian source (see solution in Fig. 7). The $H(d i v)$ error is computed versus the inverse of the cubic root of number of degrees of freedom (this quantity is equivalent to the mesh size) for affine pyramids and non-affine pyramids (Fig. 3).

Because of the presence of spurious modes, the use of Graglia's functions lead to a scheme which converge slowly and erratically. It can be seen that optimal elements require less degrees of freedom to reach a given accuracy than other elements (for $r=2$ ).

\subsection{Comparison between Hexahedral Elements}

\subsubsection{Dispersion Analysis}

A dispersion analysis will be performed for three kind of hexahedral patterns 

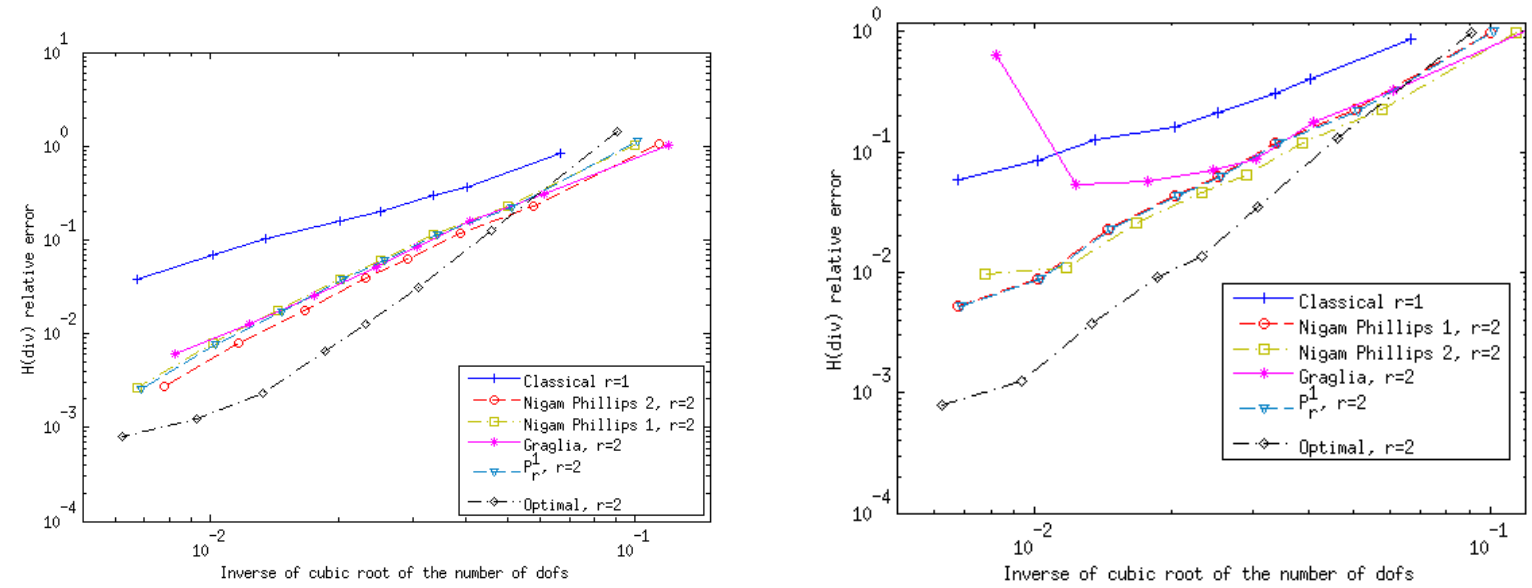

Figure 3: Relative $H($ div $)$ error for different pyramidal spaces for the source problem with only affine (left) and non-affine (right) pyramids.

1. A regular pattern made of a single cube

2. A deformed pattern made of 8 cubes, but the middle point has been moved. As a result the eight hexahedra are non-affine.

3. A split pattern made of 24 hexahedra, obtained by considering a cube containing 6 tetrahedra, and by splitting each tetrahedra into four hexahedra

These three patterns are displayed in the figure 4
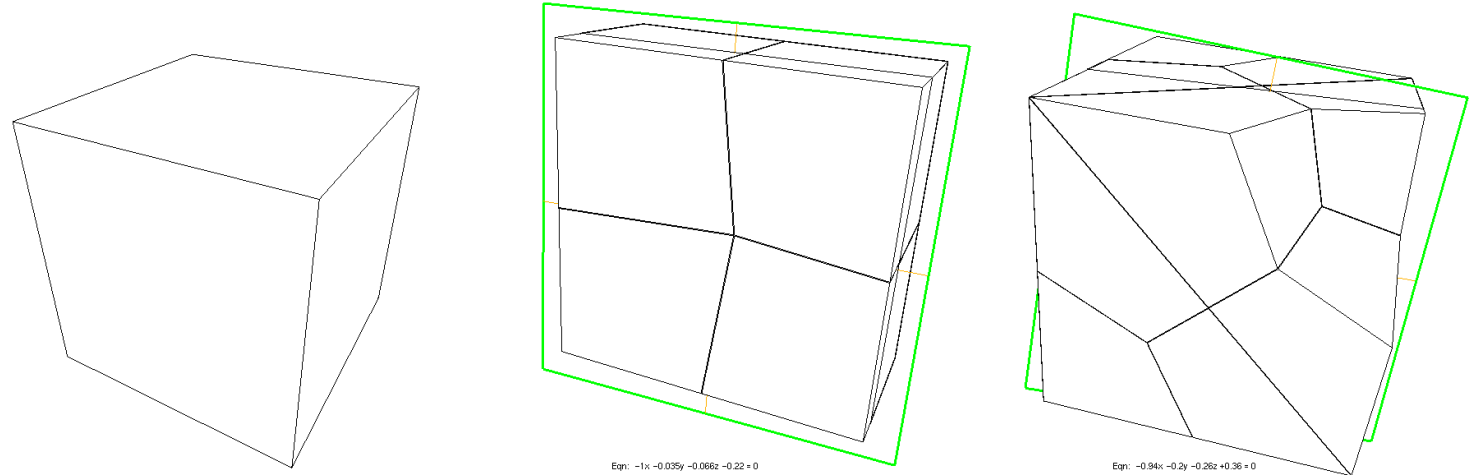

Figure 4: Patterns used to study the dispersion for hexahedral meshes. From left to right, the regular pattern, deformed pattern and split pattern.

The dispersion errors obtained for the regular pattern are displayed in Fig. 5. We observe that the dispersion error is in $O\left(h^{2 r}\right)$ for Nedelec's first family, in $O\left(h^{2 r+4}\right)$ for optimal and super-optimal finite elements. The dispersion for optimal and super-optimal spaces is exactly the same in this case, that's why we didn't display the dispersion error for super-optimal elements in the figure. The extra-power of convergence obtained for optimal elements (the dispersion error was expected to be in $O\left(h^{2 r+2}\right)$ ) is quite attractive, it should be noted that first-order optimal elements are less dispersive than third-order elements of the first kind.

The dispersion errors obtained for the deformed pattern and the split pattern are plotted in Fig. 6 .

For the deformed pattern, the observed dispersion error is in $O\left(h^{2 r-2}\right)$ for elements of the first kind, in $O\left(h^{2 r}\right)$ for optimal and super-optimal finite elements. It should be noted that optimal elements are much less dispersive than super-optimal elements for $r=1$, whereas the difference is quite small for $r=2$.

For the split pattern, the observed dispersion error is in $O\left(h^{2 r-4}\right)$ for elements of the first kind, in $O\left(h^{2 r}\right)$ for optimal and super-optimal finite elements. In that last case, optimal elements are less dispersive than super-optimal 


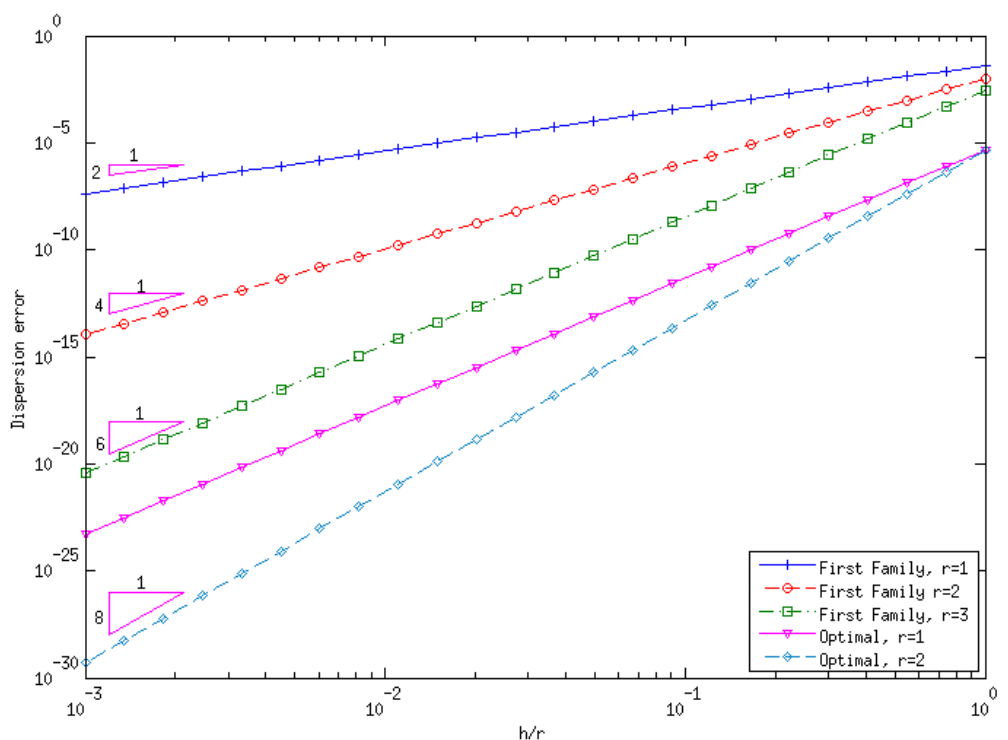

Figure 5: Dispersion error versus $h / r$ for regular patterns (hexahedral mesh).
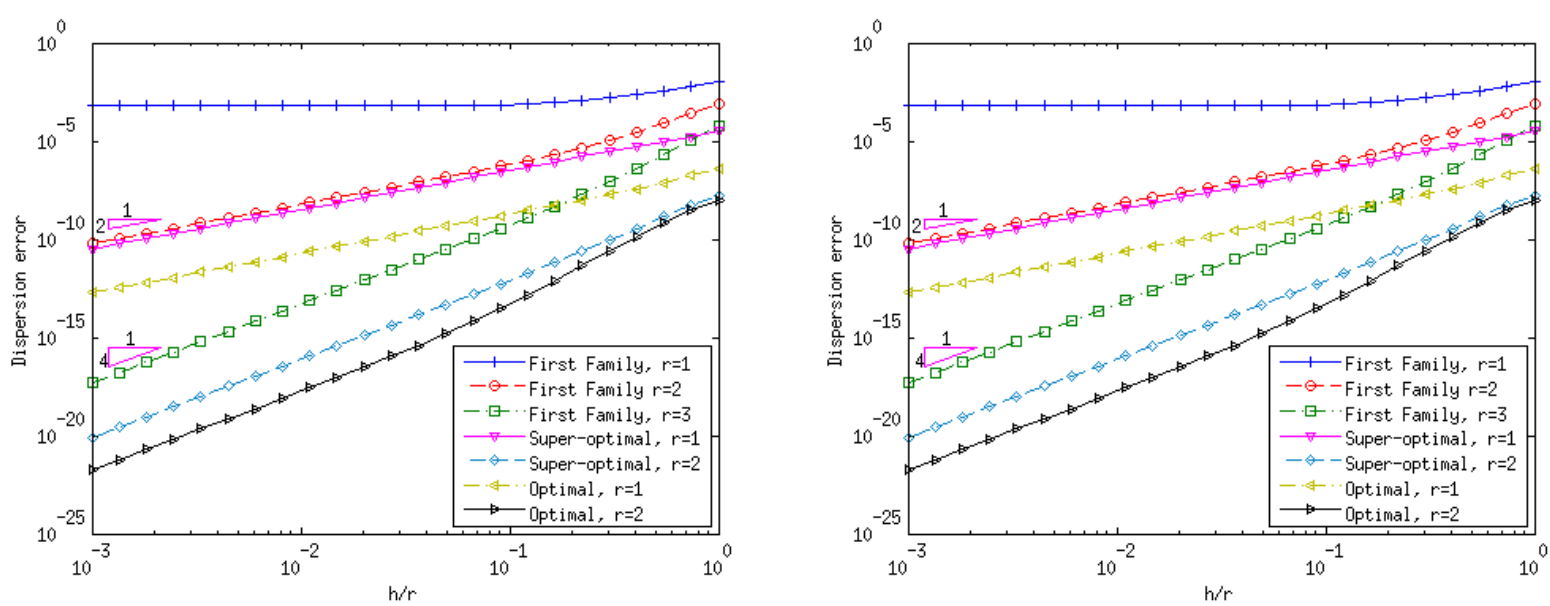

Figure 6: Dispersion error versus $h / r$ forthe deformed patterns (left) and the split patterns (right). 
elements for $r=1$, and provide the same dispersion error for $r=2$. It is interesting to see that the loss of two orders by elements of the first kind does not appear for every non-affine mesh, since the deformed meshes exhibit a loss of one order only.

\subsubsection{Convergence for the source problem}

The wave equation (1) is considered with $f$ a gaussian source oriented along $e_{x}$. The computation is performed on the cube $[-1,1]$ with homogeneous Dirichlet condition $u \cdot n=0$ on the boundary and $\omega=1.96 \pi$. The solution of this source problem is plotted in Fig. 7. The convergence in $H(d i v)$ norm is displayed for a deformed pattern
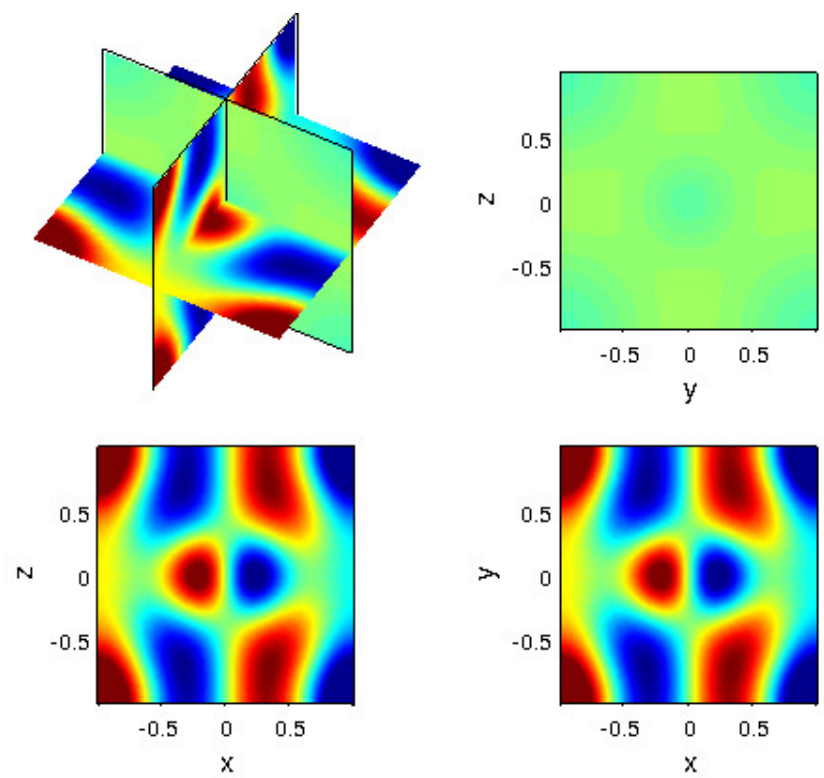

Figure 7: Divergence of $u$ for the source problem in a cube (the source being a gaussian).

and for a split pattern in Fig. 8. It is interesting to notice that for the split pattern, the first-order elements are not converging at all, the error is greater than $90 \%$ and second-order elements seem to slowly converge, we think that the convergence curve would probably stagnate for smaller values of $h$. In $x$-coordinates of those figures, the inverse of the cubic root of dofs is considered (so that it is equivalent to a mesh size $h$ ), we can compare the results for a same number of degrees of freedom. In both kinds of meshes, it appears that optimal elements require less degrees of freedom than elements of the first kind. The number of needed degrees of freedom for third-order elements of the first kind is more important than for first-order optimal elements. The difference between optimal and super-optimal elements is of relative importance for first-order approximation, and negligible for higher orders.

The wave equation is then solved for an incident wave field, and with a dielectric sphere of radius 2 :

$$
\rho=1, \quad \mu=\left\{\begin{array}{l}
2 \text { inside the sphere } \\
1 \text { outside the sphere }
\end{array}\right.
$$

The boundary $\Sigma$ is the border of the cube $[-3,3]$. The solution of this problem is plotted in Fig. 9. The meshes considered here are fully hybrid, and contain mostly hexahedra, and a small part of tetrahedra, pyramids and prisms, an example of mesh is represented in figure 10. On this test case, we have searched the needed mesh in order to obtain a $H($ div $)$ relative error equal to $1 \%$. Curved elements are used in order to obtain a good approximation of the geometry. The results are summarized in Tab. 1 . The tables are set such that we can directly compare the spaces $\hat{P}_{r}^{\text {opt }}$ and $\hat{P}_{r+1}^{1}$. It can be observed that optimal elements require less degrees of freedom than classical elements, especially for low orders. 

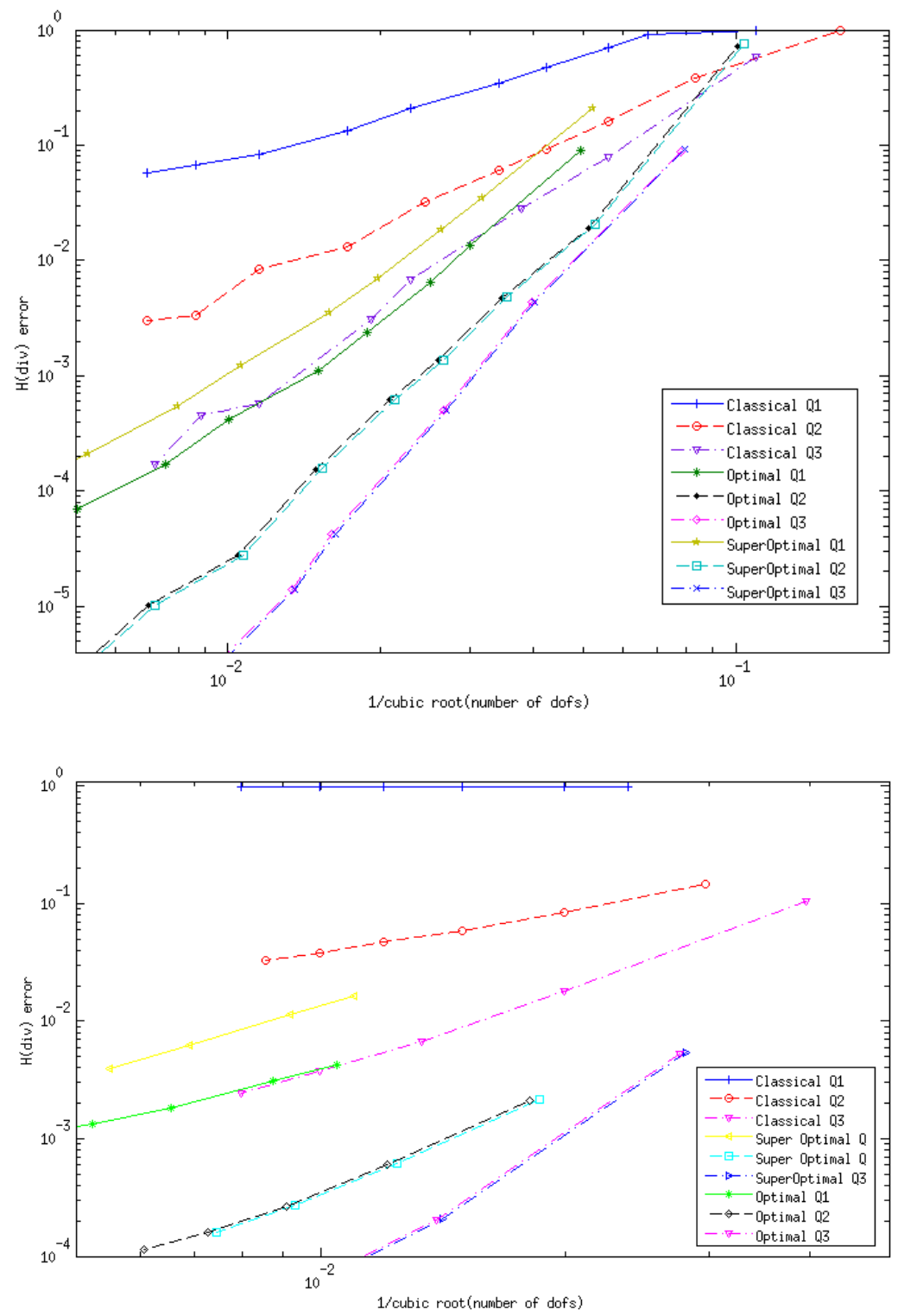

Figure 8: Numerical error in $H(d i v)$ norm versus the cubic root of number of degrees of freedom for the gaussian source in the cube. Meshes obtained with a deformed pattern (top) and with a split pattern (bottom). 

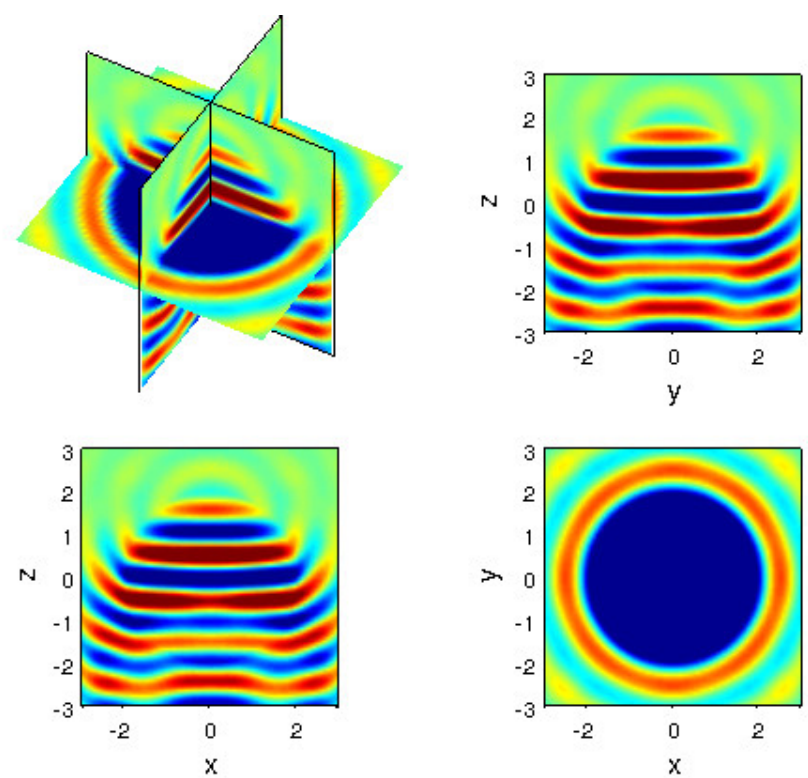

Figure 9: Divergence of $u$ of diffracted field (real part) for the sphere.

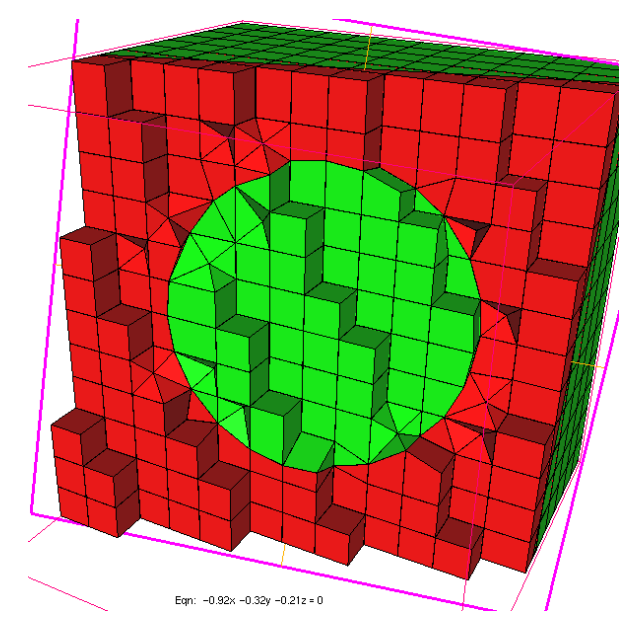

Figure 10: Hybrid mesh used for the sphere

Table 1: Stats of meshes needed to obtain a $H(d i v)$ relative error approximatively equal to $1 \%$ for the sphere. On top, elements of the first kind, on bottom optimal elements.

\begin{tabular}{|c|cccc|}
\hline Order & 2 & 3 & 4 & 5 \\
\hline Number of dofs & 5604255 & 1567977 & 528896 & 444515 \\
\hline$H($ div $)$ error & $1.34 \%$ & $0.855 \%$ & $1.04 \%$ & $0.93 \%$ \\
\hline
\end{tabular}

\begin{tabular}{|c|cccc|}
\hline Order & 1 & 2 & 3 & 4 \\
\hline Number of dofs & 1182438 & 877665 & 435488 & 471695 \\
\hline$H($ div $)$ error & $1.22 \%$ & $0.497 \%$ & $0.72 \%$ & $0.27 \%$ \\
\hline
\end{tabular}




\section{References}

[1] M. Bergot, G. Cohen, M. Duruflé, Higher-order finite elements for hybrid meshes using new nodal pyramidal elements, J. Sci. Comput 42 (3) (2010) 345-381.

[2] M. Bergot, M. Duruflé, High-order optimal edge elements for pyramids, prisms and hexahedra, J. Comp. Phys. accepted.

[3] B. Cockburn, J. Gopalakrishnan, Incompressible finite elements via hybridization.part ii: The stokes system in three space dimensions, SIAM Journal on Numerical Analysis 43 (2005) 16511672.

[4] A. Sboui, J. Jaffré, J. Roberts, A composite mixed finite element for hexahedral grids, SIAM J. Sci. Comput. (2009) $2623-2645$.

[5] J. C. Nédélec, Mixed finite elements in $\mathbb{R}^{3}$, Numer. Math. 35 (3) (1980) 315-341.

[6] J. C. Nédélec, A new family of mixed finite elements in $\mathbb{R}^{3}$, Numer. Math. 51 (1) (1986) 57-81.

[7] P. Raviart, J. Thomas, A mixed finite element method for 2nd order elliptic problem, Lecture Notes in Mathematics 606 (1977) $292-315$.

[8] R.L.Naff, T.F.Russell, J.D.Wilson, Shape functions for velocity interpolation in general hexahedral cells, Comput. Geosci. 6 (2002) 285314.

[9] D. N. Arnold, D. Boffi, R. S. Falk, Quadrilateral H(div) finite elements, SIAM J. Numer. Anal. 42 (6) (2005) $2429-2451$.

[10] R. Falk, P. Gatto, P. Monk, Hexahedral H(div) and H(curl) finite elements, ESAIM: M2AN 45 (1) (2011) 115-143.

[11] S. Owen, S. Saigal, Formation of pyramid elements for hexahedra to tetrahedra transitions, Comp. Meth. Appl. Mech. Eng. 190 (2001) $4505-4518$

[12] N. Nigam, J. Phillips, High-order finite elements on pyramids: approximation spaces, unisolvency and exactness., IMA J. of Nu. Anal. 32 (2) (2012) 448-483.

[13] N. Nigam, J. Phillips, Numerical integration for high order pyramidal finite elements, in revision.

[14] R. D. Graglia, D. R. Wilton, A. F. Peterson, I.-L. Gheorma, Higher order interpolatory vector bases on pyramidal elements, IEEE Trans. Ant. Prop. 47 (5) (1999) 775-782.

[15] P. Monk, Finite element methods for Maxwell's equations, Oxford Science Publication, 2002.

[16] L. Demkowicz, J. Kurtz, D. Pardo, M. Paszynski, W. Rachowicz, A. Zdunek, Computing With hp-Adaptive Finite Elements, Volume II, Chapman \& Hall/CRC, 2007.

[17] P. Dular, J.-Y. Hody, A. Nicolet, A. Genon, W. Legros, Mixed finite elements associated with a collection of tetrahedra, hexahedra and prisms, IEEE Trans. Mag. 30 (5) (1994) 2980-2983.

[18] P. Šolín, K. Segeth, A new sequence of hierarchic prismatic elements satisfying de rham diagram on hybrid meshes 0 (0) (2005) 1-24.

[19] V. Gradinaru, R. Hiptmair, Whitney elements on pyramids, Elec. Trans. Num. Anal. 8 (1999) 154-168. 